\title{
Strategic behavior and price discovery
}

\author{
Luis Angel Medrano* \\ and
}

Xavier Vives**

We analyze the effects of strategic behavior by a large informed trader in a price discovery process used in opening auctions in continuous trading systems. It is found that the large informed trader manipulates the market using a contrarian strategy to neutralize the effect of the trades of competitive informed agents. Furthermore, consistent with the empirical evidence available, we find that information revelation accelerates close to the opening, that the market price approaches but does not converge to the fundamental value, and that the expected trading volume displays a $U$-shaped pattern.

\section{Introduction}

- Consider a price discovery process in a financial market where traders submit orders to the system for a certain period of time before the opening (say one hour or one hour and a half) and theoretical market-clearing prices are quoted periodically as orders accumulate. Such information tâtonnement processes are used in the preopening period of continuous, computerized trading systems in several exchanges (for example, in the Paris Bourse, Toronto Stock Exchange, Bolsa de Madrid, or the Arizona Stock Exchange (AZX)). No trade is made until the end of the tâtonnement, and at any point agents may revise their orders. This preopening auction is designed to decrease the uncertainty about prices after a period without trade. ${ }^{1}$ In the Deutsche Börse with the Xetra system, there is an opening auction that begins with a call phase in which traders can enter and/or modify or delete existing orders before the (short) price determination phase. The indicative auction price is displayed when orders are executable. ${ }^{2}$ However, it has been claimed that this type of price discovery process may result in gaming and manipulation because traders can revise their orders at no cost. (See Stoll and Whaley, 1990, and Madhavan and Panchapagesan,

*Universitat Pompeu Fabra; luis.medrano@econ.upf.es.

** Institut d'Anàlisi Econòmica, CSIC; iiaed@cc.uab.es.

Partial support from DGICYT projects 93-0679 and PB98-0696 is gratefully acknowledged. We are grateful to Peter Bossaerts, Joe Harrington, Craig Holden, three anonymous referees, and seminar participants at numerous institutions for helpful comments.

${ }^{1}$ See Withcomb (1985) and Amihud and Mendelson (1987). In the NYSE the specialist provides some information to floor traders, but there is no organized information tâtonnement to set the opening price. In some circumstances the revision of orders is allowed at the opening (see Stoll and Whaley, 1990). For evidence of price discovery in the preopening at the NYSE and Nasdaq see, respectively, Madhavan and Panchapagesan (2000) and Cao, Ghysels, and Hatheway (1998).

${ }^{2}$ Otherwise the best limit/ask limit is displayed. See Xetra Market Model Release 3 at www.exchange.de. 
2000.) In Xetra the call phase "has a random end after a minimum period in order to avoid price manipulation." ${ }^{3}$

We analyze the effect of strategic behavior by a large informed agent in a stylized version of the described price discovery process. We address the possible emergence of market manipulation by the strategic informed trader, the informational efficiency of the tâtonnement in his presence, and the dynamic behavior of basic market parameters like depth, volatility, and volume. The article makes two interrelated contributions. First, it makes progress toward understanding the forces driving the pretrading period and its effectiveness as a price discovery mechanism. Second, it sheds light on contrarian behavior as a market-manipulation strategy of the insider-namely, trading against one's own information to conceal or distort the public information contained in prices in order to profit at a later date.

Biais, Hillion, and Spatt (1999) have undertaken an empirical analysis of the preopening period in the Paris Bourse. The following stylized facts emerge from their analysis. ${ }^{4}$ The preopening period is active, in particular close to the opening. In fact, the last 15 minutes before the opening (that is, 9:45 to 10:00 A.M. in the Paris Bourse) make up the most active order placement period in the day (including therefore the period with real trade). Trading at the opening amounts to about $10 \%$ of the day's total trading. About half of the preopening orders are actually executed (that is, they are serious orders), and about $60 \%$ of those are executed at the opening and not later (and, therefore, the preopening order flow is directly linked to the opening price). The average size of orders placed in the preopening period increases as we get closer to the opening. The volume of trade typically has a U-shaped form, dropping after the first round to increase sharply later when approaching the opening.

Biais, Hillion, and Spatt do not reject the hypothesis of semi-strong efficiency for prices (the "pure learning hypothesis") after 9:50. Before then, the "pure noise hypothesis" that prices do not reflect any information cannot be discarded. ${ }^{5}$ The speed of learning from prices is large in the second part of the preopening, on the order of $n^{3 / 2}$, where $n$ is the number of rounds in the tâtonnement. This means that the precision of prices grows more than linearly toward the end of the process (a price precision of the order of $n$ is associated with a learning speed of $n^{1 / 2}$ ). Furthermore, large traders place sometimes relatively unaggressive orders to limit the price impact (and therefore information revelation) and tend to modify their orders. ${ }^{6}$

The modelling of the preopening period, in which no real trade occurs until the market opens and in which traders can revise and/or cancel orders, must explain the incentives of traders to submit serious orders. However, as stated above, in the Paris Bourse a good percentage of orders seem to be "serious." Several factors may explain this fact. A principal reason may be noise in the communication channel, with the possibility of breakdown, implying that a standing order cannot be revised before the market opens or perhaps that a desired sequence of orders cannot be made effective. Obviously, the risk of communication breakdown grows larger as the opening approaches. A similar effect may be produced with a limited attention capacity and multiple matters that require attention and arrive randomly. All these considerations point toward a "deadline" effect that imposes a substantial execution risk on traders who wait until close to the opening to place an order. This deadline effect is built into the Xetra system with the random end of the call phase. ${ }^{7}$

Other built-in mechanisms in price discovery processes give advantages to traders placing orders early (and therefore contributing to price discovery). For example, the fee for submitting

\footnotetext{
${ }^{3}$ See Xetra Market Model Release 3 at www.exchange.de.

${ }^{4}$ See their article for details of the tâtonnement in the Paris Bourse, and see Biais. Hillion, and Spatt (1995) for a general analysis of trading in the Paris Bourse.

${ }^{5}$ A similar result is obtained by Sola (1999) with data from the Bolsa de Madrid.

${ }^{6}$ The fact that large agents tend to minimize the price impact of their trades has already been established in the empirical literature (see, for example, Chan and Lakosnishok, 1995 and Keim and Madhavan, 1995, 1996).

${ }^{7}$ Other incentives to submit orders may be related to sunshine trading (see Admati and Pfleiderer, 1991) or cooperative mechanisms by market makers to produce "price discovery" (Cao, Ghysels, and Hatheway (1998) find leadership patterns among market makers in the preopening at Nasdaq).

(1) RAND 2001 .
} 
an order may rise as the opening approaches (as in the Arizona Stock Exchange), or time priority advantages may be established. (See, for example, Economides and Schwartz (1995).) In the Paris Bourse, for example, there is a limited but significant role for time priority (especially since June 1995). Similarly, activity rules have been proposed in the design of electricity markets (as the California Power Exchange) to prevent gaming and encourage the convergence to an efficient outcome (Wilson, 1997).

Another, somewhat more exotic, instance in which it must be explained why agents submit orders early is parimutuel horse betting. Bettors put money on a horse and bet against each other. Investors in a winning horse keep the money invested and obtain a share of all the money invested in the losing horses (minus the racetrack's cut). During a period bets can be placed at any time until the race starts. The odds are displayed every minute. The reported (win) odds for a horse are the return (net of getting the invested money back) per dollar invested. Obviously, the only odds that matter are the final ones (computed with the total amounts wagered) when the race starts. The temporal pattern of cumulated betting on horses resembles the pattern of orders in the preopening tâtonnement, rising sharply toward the end (Camerer, 1998). However, early betting is not insignificant and seems difficult to explain, since an early bettor faces some price risk unless the bet is cancelled before the start (something that is allowed at only some racetracks). The possibility of cancellation enhances the prospect of market manipulation.

Field studies suggest that parimutuel betting is an efficient aggregator of information (see, for example, Thaler and Ziembra (1988)). That is, the odds that result from the betting are closely related to the horses' relative frequencies of winning. Favorites that pay low odds win often, while "longshots" that pay high odds win only occasionally. (A small longshot bias has been reported, however: the odds on longshots are somewhat too high.) Experimental results by Plott, Wit, and Yang (1997) are consistent with the field evidence. In their experimental design, individuals are endowed with private information and given the needed computations based on Bayes' rule. In the experiment, markets open simultaneously, odds are available every minute, and all markets are closed simultaneously at a random time within a preset interval without warning. It is found that most volume concentrates close to the first time at which the market can close. Furthermore, there is some limited evidence in the experiments of market-manipulation attempts (which impair informational efficiency). (See also Nöth and Weber (1996).)

The field experiment conducted by Camerer (1998) concludes that temporary large bets, which are cancelled before the start, move the odds of the horses but the net effect is statistically insignificant. That is, in no period (be it prebet or postbet) do other bettors respond systematically to the temporary bets of the "manipulator" with respect to a baseline of matched-pair control horses in the same race. There does not seem to be evidence of the effectiveness of manipulation in this context, despite the fact that interventions closer to the opening moved the odds more. ${ }^{8}$ The results are interpreted as the parimutuel markets being good aggregators of information.

In the present article, as in Vives (1995), we assume that at any stage in the process there is a positive probability that the market opens and trade is realized, and with the complementary probability the tâtonnement continues. It is reasonable to suppose that the probability that the market opens is increasing as the tâtonnement progresses. The assumption is somewhat crude, but it has the advantage of yielding a tractable model. ${ }^{9}$ Furthermore, in some actual markets it is descriptive. In the Xetra system of the Deutsche Börse at the opening, intraday, and closing auctions there is a call phase with a random end (after a minimum initial period). The stated objective of the random end of the call phase is to avoid price manipulation. ${ }^{10}$ Our model, despite

${ }^{8}$ As the author notes, however, the manipulation test is a test of a joint hypothesis of ignorance of the existence of a manipulator, noticeability of the bets, asymmetry of reaction to the placement and cancellation of the manipulating bets, and some specific behavioral assumptions about the population of bettors.

${ }^{9}$ The assumption is similar in spirit to the exogenous random delays in communicating offers in the model of bargaining with deadlines of Ma and Manove (1993). In this context, players begin to send offers when there is a positive probability that an offer may arrive after the deadline has expired.

${ }^{10}$ All these auctions have three phases: call, in which orders can be entered or preexisting orders modified or cancelled; price determination; and order book balancing. Volatility interruptions may occur during auctions or continuous 
being close to the Xetra call auctions, is better seen as a parable to explain some driving forces of the actual preopening price discovery process, but it does not purport to match it exactly. The model does illuminate, for example, whether and how a random opening of the markets controls price manipulation by informed large traders.

Our tâtonnement was inspired by some dynamic adjustment processes to implement rationalexpectations equilibria (Jordan, 1982, 1985, and Kobayashi, 1977). The model presented is akin to the dynamic trading model of Kyle (1985) with two variations: the addition of a competitive sector of risk-averse privately informed agents and the absence of trade unless the market opens, and this happens with a specified probability at any stage in the process. The model can also be seen as the tâtonnement in Vives (1995) with the addition of a risk-neutral large informed trader.

We obtain the following results: The strategic informed trader may have an incentive to manipulate the market at the beginning of the tâtonnement. The reason is that by doing so the strategic informed trader can keep the market price uninformative, while the probability that the market opens is low, in order to trade aggressively when the market is likely to open. The way to manipulate the market is by taking an opposite position to the competitive informed agents, who trade according to their information. In this way, if the strategic informed trader sees that the liquidation value of the asset is high relative to public information, he will place an order to sell to compensate the orders of the competitive traders who will be buying in the aggregate. The cost of manipulating the market is low when the probability that the market opens is low and this happens at the beginning of the tâtonnement. Indeed, we find that if the probability that the market opens is very low, the strategic informed trader will always manipulate the market and will make the market price almost uninformative about the liquidation value of the risky asset.

We also find that the trading intensity of the large informed trader is increasing in the probability that the market opens. We see therefore that a random opening time does not eliminate price manipulation but limits it. Indeed, for a large enough opening probability, the strategic trader does not manipulate (in the sense of trading against the information he has) the market. Without the presence of competitive informed traders, the strategic informed trader would have incentives to respond little to his information at the beginning, to keep prices uninformative, but there would be no need to manipulate the market. In any case, the informativeness of prices is kept low until the end of the tâtonnement, at which time it increases sharply.

Using simulations of the model, we obtain three important results consistent with the empirical evidence (and for a natural specification of the sequence of probabilities determining the likelihood of the market opening). First, price precision is a strictly convex function of $n$, and therefore the rate of increase of the price precision increases with $n$ in contrast with the competitive case. Furthermore, it is easy to produce estimates of the speed of learning of the order obtained by Biais, Hillion, and Spatt (1999). Information revelation accelerates as the tâtonnement progresses due to the activity of the strategic informed trader. Second, the market price does not converge to the fundamental value no matter how long the horizon of the tâtonnement. Third, for plausible parameter configurations, total expected trading volume is U-shaped. This is driven by the presence of the strategic informed trader and his manipulative trading pattern.

The three results contrast with the case where there is no strategic informed trader (Vives, 1995). Then the rate of increase of the price precision is linear, and the price does converge to the fundamental value as the horizon of the tâtonnement lengthens. The presence of the strategic informed trader sets a definite upper bound on the information content of prices. Furthermore, expected trading volume is decreasing because the volume traded by competitive agents tends to decrease.

The simulations also show that in an economy in which the strategic informed trader is smaller, he responds more to his private information, prices reveal more information and are more volatile, and there is more trading. A more competitive market is associated with improved informational efficiency and more trading. It is also worth mentioning that the competitive informed

trading when prices lie outside certain predetermined price ranges. A volatility interruption is followed by an extended call phase, which also terminates randomly.

(1) RAND 2001. 
traders benefit from the presence of the strategic informed trader, because then prices tend to be less informative and the competitive agents can profit more from their information. Conversely, the strategic informed trader would like the competitive sector to be nonexistent or as small as possible.

It is assumed that all random variables are normally distributed and that the competitive informed traders display CARA utilities. Attention is concentrated in linear equilibria. ${ }^{11}$ The model is developed under the assumption (as in Kyle (1985) and Vives (1995)) that informed agents (competitive and strategic) submit market orders. We have also checked that the general pattern of results obtained in the article holds when the competitive informed agent and the strategic informed trader submit demand schedules.

The present work is also related to the stock-price manipulation literature. This literature can be classified according to whether manipulation is based on actions that change the value (or the perceived value) of the asset, or on releasing misleading information, or purely on trade. Examples of the first type are given in Vila (1989), of the second type in Vila (1989) and Benabou and Laroque (1992), and of the third type in Hart (1977), Jarrow (1992), Allen and Gale (1992), Allen and Gorton (1992), Kumar and Seppi (1992), Fishman and Hagerty (1995), and Brunnermeier (1998). ${ }^{12}$ Our model belongs to the third class, with trade-based manipulation. In our case, however, the objective of the strategic informed trader, who has accurate information on the liquidation value of the asset (and other agents know this), is to neutralize the informative trades that competitive informed agents make. It is thus not the case, as it is in Allen and Gale (1992) or Fishman and Hagerty (1995), that an uninformed manipulator can pretend to be informed to manipulate the price and make money.

Contrarian behavior is obtained in two instances in the literature. John and Narayanan (1997) develop a variation of the model of Fishman and Hagerty (1995) in which mandatory disclosure of trades of corporate insiders gives them, under certain assumptions, incentives to manipulate the market using a contrarian strategy. ${ }^{13}$ Foster and Viswanathan (1994) provide an example of a duopoly where information has a common and a private component and where the better-informed agent tries to minimize the learning of the lesser-informed one. This market manipulation may lead to contrarian behavior by the better-informed trader if the private and common signals have very disparate realizations (something that happens with low probability and therefore does not happen on average). Finally, Hillion and Suominen (1998a) explain the incentives of brokers to manipulate the prices at the close to "look good" in front of customers. Evidence of strategic behavior at the close of the Paris Bourse is provided in Hillion and Suominen (1998b).

Our results also suggest that strategic behavior may impair the informational efficiency of market mechanisms even when attention is concentrated in the class of linear ("separating") equilibria, reinforcing the point made by Laffont and Maskin (1990), who argue that pooling equilibria may obtain in a context with multiple equilibria.

In Section 2 we present the price discovery process. Section 3 characterizes the dynamic equilibrium, and Section 4 analyzes two extreme economies, competitive and monopolistic. Section 5 studies the general model, establishes the manipulation result, and presents the simulation analysis of the dynamic behavior of the model. Section 6 contains concluding remarks. The Appendix gathers some of the proofs.

${ }^{11}$ With normal distributions it is an open question whether nonlinear equilibria exist in Kyle (1985) as well as in our model. In the Kyle model, however, when the insider submits a demand schedule, there is a unique equilibrium (linear under the normality assumption) both in static (Rochet and Vila, 1994) and dynamic (Back, 1992) settings.

${ }^{12}$ For example, Allen and Gorton (1992) explain price manipulation by an uninformed agent in the presence of asymmetries in noise trading (noise selling is more likely than noise buying) or in whether buyers or sellers are informed (with short-sale constraints, exploiting good news is easier than exploiting bad news).

${ }^{13}$ Important ingredients of their model are that agents want to trade only one unit, the fundamental value follows a two-state distribution, and market makers fix prices before seeing the order flow.

(1) RAND 2001. 


\section{A price discovery process}

- Consider a market with a single risky asset with random (ex post) liquidation value $v$ and a riskless asset with unitary return traded among noise traders, a continuum of risk-averse competitive informed agents of mass $(1-\mu)$, and a large informed trader of mass $\mu$ with the intermediation of competitive market makers.

The strategic informed trader acts strategically, that is, he takes into account the effect his demand has on prices. For simplicity, we assume that he is risk neutral and observes the liquidation value $v$ in advance. His profits when buying $\mu y$ units of the asset at price $p$ are given by $\pi=(v-p) \mu y$. His initial wealth is normalized to zero. The strategic informed trader submits a market order contingent on the information he has.

There is a continuum of competitive informed agents indexed in the interval $[\mu, 1]$ (endowed with the Lebesgue measure). Each competitive informed agent has a little piece of (private) information about the unknown $v$. The profits of agent $i$ buying $x_{i}$ units of the asset at price $p$ are given by $\pi_{i}=(v-p) x_{i}$. Informed agents are risk averse and have CARA utilities: $U\left(\pi_{i}\right)=$ $-\exp \left\{-\rho \pi_{i}\right\}$, where $\rho>0$ is the coefficient of constant absolute risk aversion. The initial wealth of informed agents is also normalized to zero. Informed agent $i$ submits a market order contingent on the information he receives.

Noise traders (in the aggregate) submit an order $u$. Market makers are risk neutral and set prices efficiently conditional on the observation of the "order flow."

The horizon is finite ( $N$ periods). Price discovery is modelled as an information tâtonnement. ${ }^{14}$ At stage $n$ there is a positive probability $\gamma_{n}$ that the market opens, the value $v$ is realized, and trade occurs (given that there has not been trade before stage $n$ ), with the complementary probability $\left(1-\gamma_{n}\right)$ that there is no trade and the tâtonnement continues. We assume that the sequence $\left\{\gamma_{n}\right\}$ is nondecreasing and, obviously, that $\gamma_{N}=1$, since at $n=N$ the market opens for sure if it has not done so before.

Suppose that at the beginning of stage $n$ the market has not opened. Then the competitive informed agents and the strategic informed trader, before knowing whether there will be trade in the period, have the opportunity to submit market orders to the competitive market-making sector. These orders supersede previous orders, which are understood to be cancelled if the market does not open. ${ }^{15}$

The strategic informed trader's information is given by $\left\{v, p^{n-1}\right\}$, where $p^{n-1}=\left(p_{1}, \ldots\right.$, $\left.p_{n-1}\right)$ is the sequence of past prices. His market order is of the type $Y_{n}\left(v, p^{n-1}\right)$. The information of competitive informed agent $i$ is given by $\left\{s_{i}, p^{n-1}\right\}$, where $s_{i}$ is his private signal about $v$, and his market order is of the type $X_{n i}\left(s_{i}, p^{n-1}\right)$. Noise traders submit the aggregate order $u_{n}$. Alternatively, we could think that noise comes from a garbled communication channel in the transmission of orders or from the aggregation procedure of the order flow. In this case, market makers have a noisy observation of the order flow. ${ }^{16}$

The order flow is then $\omega_{n}=\mu y_{n}+x_{n}+u_{n}$, where $x_{n}=\int_{\mu}^{1} X_{n i}\left(s_{i}, p^{n-1}\right) d i$ is the aggregate demand of the competitive informed agents. Competitive market makers set $p_{n}=E\left(v \mid \omega^{n}\right)$, where $\omega^{n}=\left(\omega_{1}, \ldots, \omega_{n}\right)$. That is, $p_{n}$ is equal to the expectation of $v$ conditional on public information (including the current order flow $\left.\omega_{n}\right) \cdot{ }^{17}$ If the market opens at stage $n, v$ is realized, trade occurs, and this is the end of the story. Otherwise the tâtonnement continues. All trades are notional until the market opens. Informed traders can revise their orders before the market opens. In general they will have incentives to do so once they receive more public information, since this

${ }^{14}$ This is a finite-horizon version of the tâtonnement process in Vives (1995) with the addition of a large informed trader.

${ }^{15}$ The model as stated also encompasses the case in which only the orders of noise traders are cancelled if the market does not open.

${ }^{16}$ In Gould and Verrecchia (1985), for example, the price quoted by the specialist is garbled.

${ }^{17}$ Efficient pricing would be the outcome of Bertrand competition among risk-neutral market makers who observe the order flow. 
helps them to predict better the net value $v-p$. Furthermore, the strategic informed trader may be able to manipulate the information contained in prices and may have incentives to do so.

All random variables are assumed to be normally distributed with the sequence $\left\{u_{t}\right\}$ independently and identically distributed with zero mean and variance $\sigma_{u}^{2}$. The signal $s_{i}$ is given by $s_{i}=v+\varepsilon_{i}$, where $v \sim N\left(\bar{v}, \sigma_{v}^{2}\right)$ and $\varepsilon_{i} \sim N\left(0, \sigma_{\varepsilon}^{2}\right)$ with $\operatorname{cov}\left(v, u_{n}\right)=\operatorname{cov}\left(v, i_{i}\right)=\operatorname{cov}\left(\varepsilon_{i}, u_{n}\right)=$ $\operatorname{cov}\left(\varepsilon_{i}, \varepsilon_{j}\right)=0, j \neq i$, for all $n$. That is, the liquidation value $v$, the error terms of signals, and the sequence $\left\{u_{t}\right\}$ are mutually independent.

We will use the usual convention that given $v$, the average signal of the competitive informed agents $\tilde{s}=[1 /(1-\mu)] \int_{\mu}^{1} s_{i} d i$ equals almost surely $v$ (i.e., errors cancel out in the aggregate, $\int_{\mu}^{1} \varepsilon_{i} d i=0$ ). In other words, the pooled information of informed agents reveals $v$. We can interpret the strategic informed trader of size $\mu$ as emerging from a coalition of small informed traders (of measure $\mu$ ) who decide to form a cartel of investors and pool their information.

The information tâtonnement serves the purpose of eliciting information about the fundamental value of the asset. The notional prices quoted by the market makers convey noisy information, because of noise trading, about $v$. The described process matches those cases in which the market opens at a randomly specified time (like in the Xetra auctions) and otherwise is a crude idealization of the preopening price discovery processes in the other exchange markets described in Section 1. In the latter cases the real pattern of tâtonnement from, say, 8:30 to 10:30 A.M. and opening at 10:00 A.M. could be approximated smoothly by a sequence of probabilities $\left\{\gamma_{n}\right\}$ approximating a step function with no trade before 10:00 A.M and opening at 10:00 A.M. Furthermore, the possibility of a communication breakdown at some point (with increasing probability as the opening approaches), implying that the standing order "sticks" and cannot be revised, parallels the possibility of the market's opening at any point in the process (but with increasing probability as the end of the horizon approaches). ${ }^{18}$ In consequence, a central case is when the probability $\gamma_{n}$ is of the "breakdown" type and is given by $\gamma_{n}=\gamma^{N-n}$, where $\gamma$ is an exogenous constant that lies in the interval $(0,1)$. This implies that $\gamma_{n}$ is strictly increasing and strictly convex in $n$.

For a large horizon, the probability that the market opens is very small at the beginning for any $\gamma<1$. For $\gamma$ low, the probability that the market opens, $\gamma_{n}$, stays low until the very end. For example, if $\gamma=.1$, then $\gamma_{N-2}=1 \%, \gamma_{N-1}=10 \%$, and $\gamma_{N}=100 \%$. For $\gamma$ high, the probability that the market opens approaches one in a less abrupt way. For example, if $\gamma=.7$, then $\gamma_{N-2}=49 \%, \gamma_{N-1}=70 \%$, and $\gamma_{N}=100 \%$. For $\gamma$ close to one and a short horizon, $\gamma_{n}$ stays close to one for any $n$. For a moderate-horizon $N$, it is reasonable to choose $\gamma$ not too high so that the probability of trade is low at the beginning. For example, with $\gamma=.5$ and $N=10$ we have that $\gamma_{1}=2 \%, \gamma_{5}=3 \%, \gamma_{8}=25 \%$, and $\gamma_{9}=50 \%$.

\section{Equilibrium in the dynamic market}

- We now study the dynamics of the price discovery process in which the informed agents compete with the strategic informed trader.

We restrict attention to linear equilibria. In consequence, the normality of the order flow is preserved and $p_{n}=E\left(v \mid \omega^{n}\right)$ is a sufficient statistic in the estimation of $v$ with respect to the information $\left\{\omega^{n}\right\}$. It follows that $E\left(v \mid p_{n}\right)=p_{n}$ and prices follow a martingale: $E\left(p_{n} \mid\right.$ $\left.p^{n-1}\right)=p_{n-1}$. As we shall see, it is easily shown that $p_{n}=\lambda_{n} \omega_{n}+p_{n-1}$, where as usual $\left(\lambda_{n}\right)^{-1}$ is an index of the depth of the market. The conditional volatility of prices is given by $\operatorname{var}\left\{p_{n} \mid p_{n-1}\right\}=\left(\tau_{n-1}\right)^{-1}-\left(\tau_{n}\right)^{-1}$, where $\tau_{n} \equiv\left[\operatorname{var}\left(v \mid p_{n}\right)\right]^{-1}$ is the precision of the price in the estimation of $v$ (let $\left.p_{0} \equiv \bar{v}\right){ }^{19}$

At stage $n$ a strategy for competitive agent $i$ is a function that maps his private information $s_{i}$ and the observed past prices $p^{n-1}$ into desired purchases: $X_{i n}\left(s_{i}, p_{n-1}\right)$. The agent will face the

${ }^{18}$ It must be pointed out that our model does not match exactly the communication breakdown story, since in the latter a trader has a certain individual probability of being cut off from the market, whereas in our model the market opens or not for everybody.

${ }^{19}$ The precision of a random variable $x,\left(\sigma_{x}^{2}\right)^{-1}$, will be denoted in general by $\tau_{x}$. 
following expected utility conditional on his information $\left\{s_{i}, p^{n-1}\right\}: \gamma_{n} E\left\{U\left(\pi_{i n}\right) \mid s_{i}, p_{n-1}\right\}+$ $\left(1-\gamma_{n}\right) E\left\{u_{n} \mid s_{i}, p_{n-1}\right\}$, where $\pi_{i n}=\left(v-p_{n}\right) x_{i n}, U\left(\pi_{i n}\right)=-\exp \left\{-\rho \pi_{i n}\right\}$, and $u_{n}$ is the (random) continuation utility, which, given that the agent is negligible, is independent of the current market order $x_{i n}$. The agent behaves as if the asset were to be liquidated and trade realized in the period. From the point of view of a competitive agent, the only difference between periods is in the information available. In consequence, a competitive agent will behave myopically and will optimize choosing a market order with the usual form in a CARA-normal model (the strategy is symmetric given that signals have the same precision):

$$
X_{n}\left(s_{i}, p_{n-1}\right)=\frac{E\left\{\left(v-p_{n}\right) \mid s_{i}, p_{n-1}\right\}}{\rho \operatorname{Var}\left\{\left(v-p_{n}\right) \mid s_{i}, p_{n-1}\right\}} .
$$

At stage $n$ a strategy for the strategic informed trader is a function that maps his private information $v$ and the observed past prices $p^{n-1}$ into desired purchases. He knows that the asset will be liquidated and trade realized in the period with probability $\gamma_{n}$ and with probability $\left(1-\gamma_{n}\right)$ he will obtain the continuation (expected) profit which depends on his market order in period $n$. At stage $n$ the strategic informed trader will face the following expected profit conditional on his information:

$$
E\left\{\pi_{n} \mid v, p^{n-1}\right\}=\gamma_{n} E\left\{\left(v-p_{n}\right) \mu y_{n} \mid v, p^{n-1}\right\}+\left(1-\gamma_{n}\right) E\left\{\pi_{n+1} \mid v, p^{n-1}\right\},
$$

where $E\left\{\pi_{n+1} \mid v, p^{n-1}\right\}$ is the expected continuation profit (which depends on the current and the past market orders).

Restricting attention to equilibria in linear strategies, it is possible to obtain a full characterization of equilibrium behavior in our model with risk-averse competitive agents and a large informed trader.

Proposition 1. If there exists a linear equilibrium, then it must have the following characteristics:

$$
\begin{aligned}
X_{n}\left(s_{i}, p^{n-1}\right) & =a_{n}\left(s_{i}-p_{n-1}\right), \\
Y_{n}\left(v, p^{n-1}\right) & =\alpha_{n}\left(v-p_{n-1}\right), \\
p_{n} & =\lambda_{n} \omega_{n}+p_{n-1},
\end{aligned}
$$

where $\omega_{n}=A_{n}\left(v-p_{n-1}\right)+u_{n}, \lambda_{n}=\tau_{u} A_{n} / \tau_{n}, A_{n}=\mu \alpha_{n}+(1-\mu) a_{n}, \tau_{n}=\tau_{v}+\tau_{u} \sum_{t=1}^{n} A_{t}^{2}$. At stage $n$, the strategic informed trader's expected continuation profit is given by

$$
E\left\{\pi_{n+1} \mid v, p_{n}\right\}=\mu\left(H_{n}\left(v-p_{n}\right)^{2}+\delta_{n}\right) .
$$

The constants $a_{n}, \alpha_{n}, H_{n}$, and $\delta_{n}$ are the solution to the difference equation system

$$
\begin{aligned}
a_{n} & =\left\{\rho\left[\left(\tau_{\varepsilon}\right)^{-1}+\left(\tau_{n-1}\right)^{-1}-\left(\tau_{n}\right)^{-1}\right]\right\}^{-1} \\
\alpha_{n} & =\left\{\left[1-(1-\mu) \lambda_{n} a_{n}\right] /\left[2 \mu \lambda_{n}\right]\right\}\left\{\left[\gamma_{n}-2\left(1-\gamma \mu \lambda_{n} H_{n}\right] /\left[\gamma_{n}-\left(1-\gamma \mu \lambda_{n} H_{n}\right]\right\}\right.\right. \\
H_{n} & =\left(1-\lambda_{n+1} A_{n+1}\right)\left[\gamma_{n+1} \alpha_{n+1}+\left(1-\gamma_{n+1}\right) H_{n+1}\left(1-\lambda_{n+1} A_{n+1}\right)\right] \\
\delta_{n} & =\left(1-\gamma_{n+1}\right)\left[\delta_{n+1}+\left(\lambda_{n}\right)^{2} H_{n+1} / \tau_{u}\right],
\end{aligned}
$$

subject to the boundary conditions $H_{N}=0, \delta_{N}=0,2 \mu \alpha_{N} \lambda_{N}=\left[1-(1-\mu) \lambda_{N} a_{N}\right]$, and the second-order conditions $\lambda_{n} \mu\left[\gamma_{n}-\left(1-\gamma_{n}\right) \mu \lambda_{n} H_{n}\right]>0$ for all $n=1,2, \ldots, N$.

Corollary 1. At a linear equilibrium the following inequalities hold for all $n$ : $0<a_{n}<\tau_{\varepsilon} / \rho$, $A_{n}>0, \lambda_{n}>0,0<\mu \lambda_{n} H_{n}<\gamma_{n} /\left(1-\gamma_{n}\right), 0<\left[1-(1-\mu) \lambda_{n} a_{n}\right]<1$, and $0<\left[1-\lambda_{n} A_{n}\right]<1$.

Proof. It is a modification of the arguments in Kyle (1985) and Vives (1995). See the Appendix. 
To show existence and uniqueness of the linear equilibrium in the general model is quite a cumbersome task. ${ }^{20} \mathrm{We}$ conjecture that the linear equilibrium exists and is unique for all parameter configurations. The conjecture is based on the fact that the result holds when $\mu=0$ (Vives, 1995), when $\mu=1$ (see Section 5), and also when $N=2$ and $\gamma_{1}$ is close to zero. The systematic simulations performed in a wide range of parameter values have always produced a unique (linear) equilibrium (see Section 6).

We have assumed that informed traders use market orders. However, when the strategic informed trader and the competitive informed agents use demand schedules (demand functions) as strategies, linear equilibria have a very similar characterization. A difference is that with demand schedules the competitive traders set the same (positive) trading intensity at any period, $X_{n}\left(p_{n} ; s_{i}, p^{n-1}\right)=a\left(s_{i}-p_{n}\right)$, where $a=\rho^{-1} \tau_{\varepsilon}$. This is so because now that competitive traders can condition on the current price, the conditional price volatility does not influence their trading intensity. In this case it is possible to establish existence and uniqueness in the class of linear equilibria (see Proposition A1 in the Appendix for a characterization of the equilibrium with demand schedules). ${ }^{21}$ The model with market orders allows for richer dynamics due to the evolving strategy of competitive informed traders.

The question arises about whether the strategic informed trader may have incentives to introduce noise in his order. As in Kyle (1985), it does not pay to introduce noise. The reason is that the strategic informed trader is optimizing at any stage against a fixed conjecture on the behavior of market makers, that is, a fixed lambda. For a given market depth, it is optimal not to introduce noise in the order, since the only effect of placing a noisy order is just to distort trade from its optimal level given $v$. Put in other words, suppose there is an equilibrium with positive noise added to the strategic informed trader's market order at some stage. Then it pays the strategic informed trader to reduce the noise to zero, because this deviation is unobservable, and trade according to his information $v$.

Suppose that the strategic informed trader in period $n$ can add to his order normally distributed noise $\eta_{n}$ uncorrelated with all other random variables, with mean zero and variance $\sigma_{\eta_{n}}^{2}$. The strategic informed trader at stage $n$ draws a realization of $\eta_{n}$ and places the order $\mu\left(Y_{n}\left(v, p_{n-1}\right)+\right.$ $\eta_{n}$ ). The other agents do not observe $\sigma_{\eta_{n}}^{2}$ but have a conjecture (in equilibrium, correct) about the strategic informed trader's choice of $\sigma_{\eta_{n}}^{2}$. At stage $N$ the optimal level of added noise is zero because it cannot affect market depth (derived from the fixed conjecture of market makers) and it distorts trading. The reasoning then applies to stage $N-1$ and so on. The expected profit of the strategic informed trader in period $n$ can be written as $\phi\left(v, p_{n-1}\right)-\lambda_{n} \mu\left\{\gamma_{n}-\left(1-\gamma_{n}\right) \mu \lambda_{n} H_{n}\right\} \sigma_{\eta_{n}}^{2}$, where $\phi$ is a linear function of $v$ and $p_{n-1}$ and the parameters $\lambda_{n}$ and $H_{n}$ are as in Proposition 1 . From the second-order condition we have that $\gamma_{n}-\left(1-\gamma_{n}\right) \mu \lambda_{n} H_{n}>0$ and therefore the optimal amount of noise is $\sigma_{\eta_{n}}^{2}=0$.

For further reference, we now characterize trading volume. We define the total volume traded at stage $n$, which will be denoted by $T V_{n}$, as the sum of the absolute values of the demands coming from the different agents in the model divided by 2 . That is, the expectation of the total volume traded at stage $n$ is given by

$$
E T V_{n}=\left\{E\left[\int_{\mu}^{1}\left|x_{i n}\right| d i\right]+\mu E\left|y_{n}\right|+E\left|\omega_{n}\right|+E\left|u_{n}\right|\right\} / 2,
$$

where $\int_{\mu}^{1}\left|x_{i n}\right| d i, x_{i n}=X_{n}\left(s_{i}, p_{n-1}\right)$ is the volume traded by the continuum of informed agents, $\mu\left|y_{n}\right|, y_{n}=Y_{n}\left(v, p_{n-1}\right)$ is the volume traded by the strategic informed trader, $\left|\omega_{n}\right|$ is the volume

${ }^{20}$ To find the equilibrium, we have to solve a difference equation system with $N$ periods and 2 unknowns in each period. This is complicated because the system cannot be iterated backward as in Kyle (1985) or Holden and Subrahmanyam (1992) or forward as in Vives (1995).

${ }^{21}$ The proof of existence and uniqueness with demand schedules follows the same logic as in Kyle (1985). That is, we can find a way to iterate the dynamic equation system backward. The reason is that the responsiveness to signals of the competitive informed traders is constant through time.

(c) RAND 2001. 
traded by the market makers, and the counterpart of the order flow $\omega_{n}=\int_{\mu}^{1} x_{i n} d_{i}+\mu y_{n}+u_{n}$, and $\left|u_{n}\right|$ is the trading volume coming from the noise traders. Note that because of symmetry, the expected aggregate volume traded by informed agents is $E\left[\int_{\mu}^{1}\left|x_{i n}\right| d i\right]=(1-\mu) E\left|x_{i n}\right|$. The behavior of the total trading volume is driven by the behavior of the volume traded by informed (competitive plus strategic) agents. The following lemma provides the equilibrium expressions of trading volume.

Lemma 1. In equilibrium,

$$
\begin{aligned}
E\left|x_{i n}\right| & =(2 / \pi)^{1 / 2} a_{n}\left(1 / \tau_{\varepsilon}+1 / \tau_{n-1}\right)^{1 / 2}, \\
E\left|y_{n}\right| & =(2 / \pi)^{1 / 2}\left(\left(\alpha_{n}\right)^{2} / \tau_{n-1}\right)^{1 / 2}, \\
E\left|\omega_{n}\right| & \left.=(2 / \pi)^{1 / 2}\left(\left(\sigma_{u}\right)^{2}+\left(A_{n}\right)^{2} / \tau_{n-1}\right)\right)^{1 / 2},
\end{aligned}
$$

and

$$
\begin{gathered}
E T V_{n}=(1 / 2 \pi)^{1 / 2}\left\{(1-\mu) a_{n}\left(1 / \tau_{\varepsilon}+1 / \tau_{n-1}\right)^{1 / 2}+\mu\left(\left(\alpha_{n}\right)^{2} / \tau_{n-1}\right)^{1 / 2}\right. \\
\left.+\left(\left(\sigma_{u}\right)^{2}+\left(A_{n}\right)^{2} / \tau_{n-1}\right)^{1 / 2}+\sigma_{u}\right\} .
\end{gathered}
$$

Proof. See the Appendix.

The study of the dynamics of the market parameters of interest, like the responsiveness of agents to information, market depth, price precision, volatility, and trading volume, is complex. Before tackling the general case, we will begin by examining the properties of two extreme cases: The competitive economy $(\mu=0$, as in Vives (1995)) and the monopolistic economy where the unique informed agent is a large informed $\operatorname{trader}(\mu=1)$.

\section{Two extreme economies: Competitive and monopolistic}

- Let us start with the case in which there is no strategic informed trader $(\mu=0)$. The following comes from Proposition 5.1 in Vives (1995): At the unique linear equilibrium, as $N$ tends to infinity, $a_{N}$ converges monotonically from below to $\left(\rho \sigma_{\varepsilon}^{2}\right)^{-1}, \tau_{N}$ and $\lambda_{N}^{-1}$ tend to infinity at a rate of $N, \operatorname{var} p N$ converges monotonically from below to $\sigma_{v}^{2}$, and $\operatorname{var}\left\{p_{N} \mid p_{N-1}\right\}$ tends to zero, $E\left|x_{N i}\right|$ converges from above to $(2 / \pi)^{1 / 2}\left(\rho \sigma_{\varepsilon}\right)^{-1}$ and $E T V_{N}$ converges from above to $(2 / \pi)^{1 / 2}\left(\left(\rho \sigma_{\varepsilon}\right)^{-1}+2 \sigma_{u}\right)$.

At the equilibrium, an informed competitive agent buys or sells according to whether the private signal received is larger or smaller than the public signal. The dynamics of the market are explained as follows. As $N$ grows, prices become more informative about $v$ in a linear way $\left(\tau_{N}\right.$ increases as $N$ does). The competitive market-making sector increases the depth of the market, and $\lambda_{N}^{-1}$ grows also at the rate of $N$. The conditional variance of prices decreases and induces each informed agent to respond more to his information. The volatility of price quotations increases, since they incorporate more information, and the indicative volume of trade of informed agents decreases, since their information advantage with respect to the market makers disappears as prices become more informative. In fact, the aggregate volume of trade of informed agents against market makers $E\left|\tilde{x}_{N}\right|$, where $\tilde{x}_{N}=\int_{0}^{1} X_{n}\left(s_{i}, p_{n-1}\right) d i$, equals $(2 / \pi)^{1 / 2} a_{N}\left(1 / \tau_{N-1}\right)^{1 / 2}$ and tends to zero with $N$. In the limit, informed agents lose all information advantage.

From the fact that the precision of prices grows linearly with $N$, it is immediate that $p_{N}$ converges to $v$ at a rate of $1 / \sqrt{N}$ (more precisely, it can be shown that $\sqrt{N}\left(p_{N}-v\right)$ converges in distribution to $\left.N\left(0, \sigma_{u}^{2} \rho^{2} \sigma_{\varepsilon}^{4}\right)\right)$

Let us now compare the purely monopolistic version of the model where the unique informed agent is a large informed trader (that is, $\mu=1$ ) with the competitive economy $(\mu=0)$. Proposition 2 is a specialization of Proposition 1 letting $\mu=1$ and showing the existence and uniqueness of a linear equilibrium.

(c) RAND 2001 
Proposition 2. If $\mu=1$, there is a unique linear equilibrium. It is given by

$$
\begin{aligned}
Y_{n}\left(v, p^{n-1}\right) & =\alpha_{n}\left(v-p_{n-1}\right), \\
E\left\{\pi_{n+1} \mid v, p^{n}\right\} & =H_{n}\left(v-p_{n}\right)^{2}+\delta_{n},
\end{aligned}
$$

and

$$
p_{n}=\lambda_{n} \omega_{n}+p_{n-1},
$$

where

$$
\omega_{n}=y_{n}+u_{n}, \quad \lambda_{n}=\tau_{u} \alpha_{n} / \tau_{n}, \quad \tau_{n}=\tau_{v}+\tau_{u} \sum_{t=1}^{n} \alpha_{t}^{2} \quad \text { for } n=1,2, \ldots, N
$$

The constants $\alpha_{n}, H_{n}$, and $\delta_{n}$ are the unique solution to the difference equation system

$$
\begin{aligned}
\alpha_{n} & =\left(1 /\left(2 \lambda_{n}\right)\right)\left(\left[\gamma_{n}-2 \lambda_{n} H_{n}\left(1-\gamma_{n}\right)\right] /\left[\gamma_{n}-\lambda_{n} H_{n}\left(1-\gamma_{n}\right)\right]\right) \\
H_{n} & =\left(1-\lambda_{n+1} \alpha_{n+1}\right)\left[\gamma_{n+1} \alpha_{n+1}+\left(1-\gamma_{n+1}\right) H_{n+1}\left(1-\lambda_{n+1} \alpha_{n+1}\right)\right] \\
\delta_{n} & =\left(1-\gamma_{n+1}\right)\left[\delta_{n+1}+H_{n+1}\left(\lambda_{n}\right)^{2} / \tau_{u}\right],
\end{aligned}
$$

subject to the boundary conditions $A_{N}=0, \delta_{N}=0, a_{N} \lambda_{N}=1 / 2$, and the second-order conditions $\lambda_{n}\left[\gamma_{n}-\left(1-\gamma_{n}\right) \lambda_{n} H_{n}\right]>0$ for all $n=1,2, \ldots, N$.

Lemma A1 in the Appendix provides an iterative method to compute the equilibrium. It follows from Lemma A1 that $\tau_{n} / \tau_{v}=2^{n} \prod_{t=1}^{n}\left[1-\lambda_{t} H_{t}\left(1-\gamma_{t}\right) / \gamma_{t}\right]$ and that the sequence $\left\{\lambda_{t} H_{t}\right\}$ is determined by the sequence $\left\{\lambda_{t}\right\}$. Therefore, the sequence $\left\{\tau_{n} / \tau_{v}\right\}$ only depends on the sequence $\left\{\lambda_{n}\right\}$. This means in particular that price precision and market depth are independent of noise trading. As in Kyle (1985), the result is a consequence of the risk neutrality of the strategic informed trader. It also follows that $2 \leq \tau_{n} / \tau_{v} \leq 2^{n}$.

The strategic informed trader trades according to $Y_{n}\left(v, p^{n-1}\right)=\alpha_{n}\left(v-p_{n-1}\right)$. At stage $n$, if he places a market order and there is no trade, his future profit will decrease because of the information revealed to the market makers. But if he does not submit any order and trade is consummated, his future profit will be zero because $v$ will have been revealed. It never pays to set a negative trading intensity $\alpha_{n}$. Indeed, $\alpha_{n}<0$ is dominated by $\alpha_{n}=0$ : If there is trade, with $\alpha_{n}<0$ the strategic informed trader makes negative profits while it makes zero with $\alpha_{n}=0$; if there is no trade, with $\alpha_{n}=0$ no information is revealed to the market makers while with $\alpha_{n}<0$ some information is revealed. In fact, when $\gamma_{n}=0$ it is optimal not to trade (and set $\alpha_{n}=0$ ), since the market will not open. The optimal market order, which balances the two effects, implies a trading intensity that is lower than in the one-shot model where there is trading with probability one. Moreover, it seems intuitive that $\alpha_{n}$ should be increasing in the probability $\gamma_{n}$ (this is confirmed by the simulations below). In the one-shot model of Kyle (1985), $\lambda_{1} \alpha_{1}=1 / 2$. In our monopolistic economy, $\lambda_{n} \alpha_{n}<1 / 2$ for all $n<N$ and $\lambda_{N} \alpha_{N}=1 / 2$ (since at $t=N$ the model becomes static, as in Kyle). This means that the large informed agent refrains from trading too aggressively because there is a positive probability that there is no trade.

An important result is that for the central case where $\gamma_{n}=\gamma^{N-n}$, and in contrast to the competitive economy, no matter how long the horizon, the price precision is bounded above (and the bound depends only on the parameter $\gamma$ ). In this case the monopolistic strategic informed trader prevents the full revelation of $v$ no matter how many rounds the tâtonnement has. The following corollary states the result.

Corollary 2. If $\gamma_{n}=\gamma^{N-n}$ for any given $\gamma$, then there is $\bar{N}$ such that for $N>\bar{N}$, increases in $N$ just move the schedule $\tau_{n}$ (as a function of $n$ ) to the right. In particular, $\tau_{N}=\tau_{\bar{N}}$ for $N>\bar{N}$. Let $\bar{\tau}(\gamma)$ be the limit value of the price precision. Then $\bar{\tau}(\gamma) / \tau_{v}$ is increasing, convex with $\bar{\tau}(0)=0$, and tends to infinity as $\gamma$ tends to one. 
FIGURE 1

RESPONSIVENESS TO PRIVATE INFORMATION

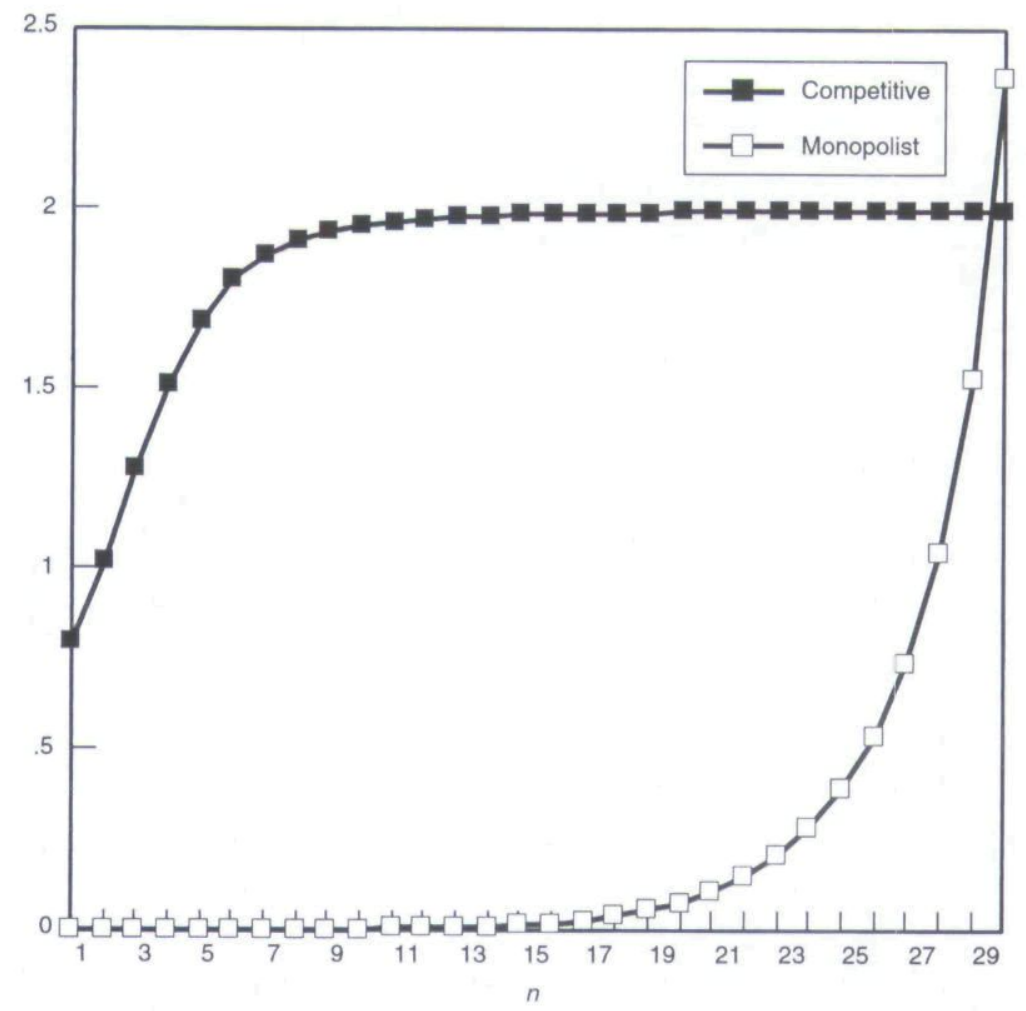

The proof of the corollary follows immediately from Proposition 2 and Lemma A1. The function $\bar{\tau}(\gamma)$ is computable. For example, $\bar{\tau}(.4)=2.8 \tau_{v}$ and $\bar{N}(.4)=10, \bar{\tau}(.6)=5.4 \tau_{v}$ and $\bar{N}(.6)=18, \bar{\tau}(.8)=50.6 \tau_{v}$ and $\bar{N}(.8)=49, \bar{\tau}(.9)=6086.2 \tau_{v}$. However, it is easy to construct sequences of probabilities $\gamma_{n}$ such that $\bar{\tau}$ is infinite. Indeed, just let, for all $n, \gamma_{n}$ be close to one. For example, $\gamma_{n}=\gamma_{n-1}+(.1 / N)$ is increasing with $\gamma_{n} \geq .9$, and $\tau_{N}$ grows exponentially with $N$. This type of probabilities sequence is not reasonable for our purposes, given that there is a very high probability that the market opens in every period. The key property for the result in the corollary to hold is that the probability that the market opens, $\gamma_{n}$, tends to zero as the number of rounds until the opening $N-n$ tends to infinity.

We shall now present a simulation analysis and discussion of the main properties of the equilibrium of the monopolistic version of the model, comparing it with the competitive version and assuming the same time horizon in both cases.

In the numerical examples in Figures 1-3, it is assumed that $\gamma_{n}=(\gamma)^{N-n}, N=30, \sigma_{v}^{2}=$ $\sigma_{u}^{2}=1, \gamma=.7, \rho=2$, and $\sigma_{\varepsilon}^{2}=.25$. Here are the results we obtain.

Responsiveness to private information. In both the competitive and the monopolistic equilibria, the responsiveness to private information increases monotonically with $n$ (see Figure 1). For $n$ small, the strategic informed trader's responsiveness to private information $\alpha_{n}$ is very close to zero, since the probability that there will be trade is low at those stages. In the competitive equilibrium, the aggregate responsiveness to private information $a_{n}$ increases to $a=\tau_{\varepsilon} / \rho$. The response of the strategic informed trader may overtake that of the competitive agents close to the end of the horizon, provided the private signals of the competitive agents are not very precise $\left(\tau_{\varepsilon}\right.$ low).

Informativeness of prices. In the competitive economy, $\tau_{n}$ increases monotonically at a rate of $n$. In the monopolistic equilibrium, $\tau_{n}$ is also monotonically increasing, but the rate of (- RAND 2001 . 
FIGURE 2

INFORMATIVENESS OF PRICES

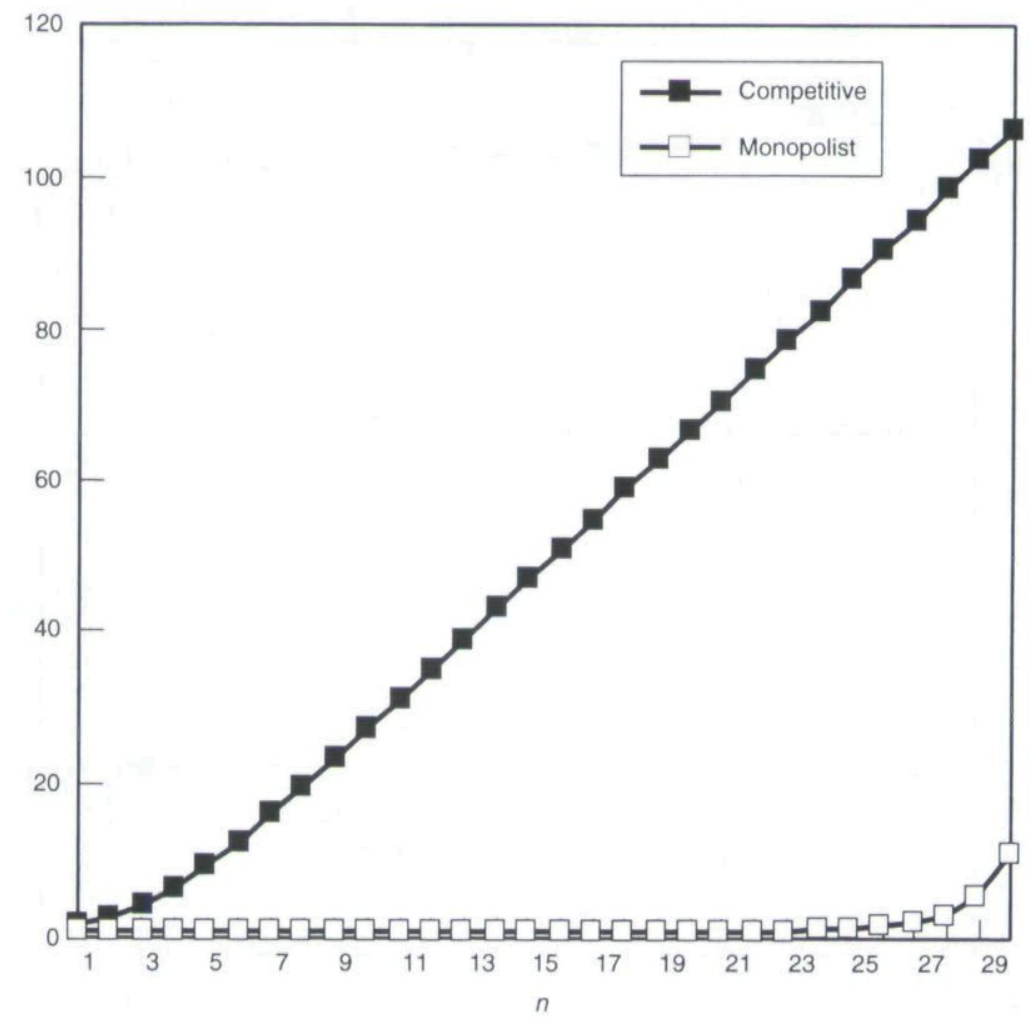

increase accelerates close to the opening (see Figure 2). In general, the informativeness of prices is higher in the competitive equilibrium for $n$ small, because at those rounds of the tâtonnement process the monopolist strategic informed trader is very reluctant to submit orders. But for $n$ close to $N$, when the probability that there will be trade is relatively high, the informativeness of prices may be higher in the monopolistic equilibrium. This is more likely to happen if the noise in the private signals of the agents in the competitive economy is high. For $N$ large, however, we know that the price precision in the competitive economy must dominate the one in the monopolistic economy, since the former grows without bound while the latter is bounded.

Market depth. In the competitive equilibrium, the depth of the market $\left(\lambda_{n}\right)^{-1}$ tends to infinity at a rate of $n$. In the monopolistic equilibrium, the market depth in general decreases during the first rounds of the tâtonnement process and then increases as the probability that there will be trade tends to one. If $n$ is small, the probability that there will be trade is relatively low. As a consequence, the strategic informed trader's trading intensity $\left(\alpha_{n}\right)$ is low, and the market is quite deep because the order flow is likely to reflect the demand of the noise traders. In this case the market makers are willing to trade because the adverse selection problem they face is not severe. As $n$ increases, the strategic informed trader behaves more aggressively (because of the higher probability of trading), the order flow is more likely to reflect the demand of the strategic informed trader, the market makers are less willing to trade, and market depth decreases. Finally, if $n$ is large and close to $N$, the market makers have a very good estimate of $v$ because of the success of the tâtonnement process, and the strategic informed trader's informational advantage is small. ${ }^{22}$

${ }^{22}$ An increase in $\alpha_{n}$ has two contradictory effects on market depth. The first is positive by making the order flow more informative about $v$, which tends to reduce the informational disadvantage of the market makers. The second is 


\section{FIGURE 3}

TOTAL EXPECTED TRADING VOLUME

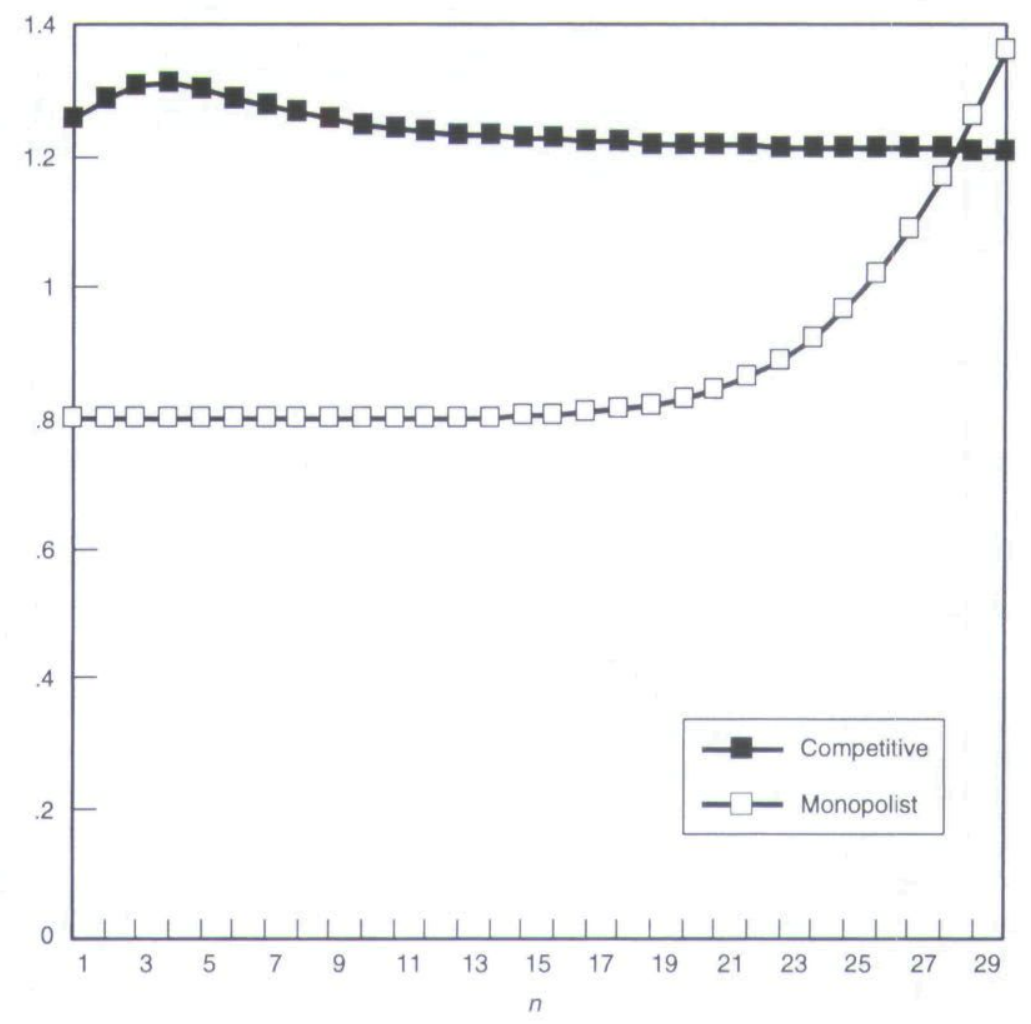

Unconditional volatility of prices. In both the competitive and the monopolistic economies, the unconditional volatility of prices var $p_{n}$ increases monotonically toward $\sigma_{v}^{2}$. In the competitive economy, however, $\operatorname{var} p_{n}$ gets close to $\sigma_{v}^{2}$ in the first few rounds of tâtonnement but at the same time is close to zero in the monopolistic economy (because the market depth is extremely high). ${ }^{23}$

Expected trading volume. In the competitive economy, the expected volume traded by informed agents is decreasing for $n$ large. On the contrary, the expected volume traded by the strategic informed trader in the monopolistic economy increases monotonically. The rate of increase is very low for $n$ small, and then it accelerates as $n$ gets close to $N$ (and as the probability that there will be trade approaches one). This qualitative feature of the monopolist equilibrium has been found to be robust to wide parameter ranges (provided that the sequence of probabilities $\left\{\gamma_{n}\right\}$ has the form proposed, $\left.\gamma_{n}=\gamma^{N-n}\right){ }^{24}$ It should be clear that

$$
E\left|y_{n}\right|=\left(\frac{2}{\pi}\right)^{1 / 2}\left[\alpha_{n}^{2} /\left(\tau_{n-1}\right)\right]^{1 / 2}
$$

negative because the order flow is more likely to reflect the demand of the strategic informed trader, which tends to make more severe the adverse selection problem faced by the market makers. The second (first) effect dominates when $\alpha_{n}$ and $n$ are small (large).

23 While it is clear that the volatility of prices is higher in the competitive equilibrium for $n$ small, this volatility may become larger in the monopolist equilibrium for $n$ close to $N$ and $N$ not too large. (This is more likely to happen if the noise in the private signals of the agents in the competitive economy is high.)

${ }^{24}$ The result depends on the form of the sequence of probabilities $\left\{\gamma_{n}\right\}$. For example, if the sequence is constant ( $\gamma_{n}=\gamma$ for all $n$ except $\gamma_{N}=1$ ) and $N$ is large, the expected volume traded by the monopolist informed trader may decrease for $n$ small and then increase as $n$ gets close to $N$. 
will be increasing if the rate of increase of $\alpha_{n}$ is sufficiently high, even though the informativeness of prices $\tau_{n-1}$ increases with $n$ as well.

The volume traded by informed agents drives the expectation of the total trading volume. This decreases to a constant $\left((2 / 2 \pi)^{1 / 2}\left\{1 /\left(\sigma_{\varepsilon} \rho\right)+2 \sigma_{u}\right\}\right)$ in the competitive model, while it increases monotonically in the monopolistic model. But since the volume traded by noise traders is constant in both models, the rate of increase of the total volume as $n$ gets close to $N$ is lower than the rate of increase of the volume traded by the monopolistic informed agent (see Figure 3).

\section{The dynamics in the general case: market manipulation}

- The most remarkable property of the equilibrium strategies (given in Proposition 1) when the strategic informed trader and the competitive informed sector coexist is that the strategic informed trader's responsiveness to private information may be negative for $n$ not too close to the end of the horizon. A negative response to private information may be interpreted as an attempt to manipulate the market. This is so because the strategic informed trader does something that is opposite to what his private information suggests, namely, at stage $n$ to buy when $v<p_{n-1}$ and to sell when $v>p_{n-1}$. Indeed, the strategic informed trader's demand is given by $\mu Y_{n}\left(v, p^{n-1}\right)=$ $\mu \alpha_{n}\left(v-p_{n-1}\right)$, so that if $\alpha_{n}<0$, the strategic informed trader's demand is negative if and only if $v>p_{n-1}$. That is, if $\alpha_{n}<0$, the strategic informed trader submits a sell order when the liquidation value of the asset is "high" and the expected return conditional in his information is positive. The purpose of doing so is to manipulate the informativeness of prices. It is an attempt to counteract the information incorporated in prices arising from the demands of the competitive informed agents. Indeed, if prices are very informative with a few rounds of tâtonnement, due to the activity of the competitive informed agents, then the strategic informed trader's opportunities to make a killing are greatly diminished. In general, the more information market makers have, the more (informationally) efficient the price is and the lower the speculative profits of informed traders are.

How can the strategic informed trader manipulate the informativeness of prices (and the depth of the market)? The informativeness of the price and the depth of the market depend on the average of the trading intensities of the strategic and the competitive informed agents, $A_{n}=\left[\mu \alpha_{n}+(1-\mu) a_{n}\right]$. Indeed, $\tau_{n}=\tau_{n-1}+\tau_{u}\left(A_{n}\right)^{2}$ and $\left(\lambda_{n}\right)^{-1}=A_{n}+\left(\tau_{n-1} /\left(\tau_{u} A_{n}\right)\right)$. The competitive informed agents will set a positive trading intensity provided their signals have positive precision, $\tau_{\varepsilon}>0$. By setting a negative $\alpha_{n}$, the strategic informed trader can decrease $A_{n}$. However, it never pays the strategic informed trader to let $A_{n}<0$. Indeed, the aggregate response to private information $A_{n}$ is always nonnegative in equilibrium. To let $A_{n}<0$, the strategic informed trader should choose $\alpha_{n}<-(1-\mu) a_{n} / \mu$. But choosing $\alpha_{n}<-(1-\mu) a_{n} / \mu$ is worse than choosing $\alpha_{n}=-(1-\mu) a_{n} / \mu$, since the expected loss in case trade is realized would be higher, and the future expected profit in case there is no trading would be lower (because if $\alpha_{n}<-(1-\mu) a_{n} / \mu$, the price does reveal some information, while if $\alpha_{n}=-(1-\mu) a_{n} / \mu$, it does not).

When will the strategic informed trader manipulate the market? We shall see that if at stage $n \gamma_{n}$ is sufficiently low, $\alpha_{n}<0$. In particular, if $\gamma_{n}=0$, then $\alpha_{n}=-(1-\mu) a_{n} / \mu$ and $A_{n}=0$. If $a_{n}>0$, then $\alpha_{n}<0 .{ }^{25}$ Indeed, if the probability of trade being realized $\left(\gamma_{n}\right)$ is zero, the strategic informed trader's optimal response is to choose a trading intensity $\alpha_{n}$ such that the price $p_{n}$ is uninformative $\left(A_{n}=\lambda_{n}=0\right.$ and $\left.p_{n}=p_{n-1}\right)$. When there is no danger of the market opening, the strategic informed trader trades in a way that no information is revealed by neutralizing the response of competitive informed agents. Given that $A_{n}=\mu \alpha_{n}+(1-\mu) a_{n}$, with $a_{n}>0$ and $0<\mu<1$, this is done by setting $\alpha_{n}=-[(1-\mu) / \mu] a_{n}<0 .{ }^{26}$ It is plausible then to expect

${ }^{25}$ Obviously, if $\gamma_{n}=0$ for $n<N$, in equilibrium, the competitive agents may put any weight on their information.

${ }^{26}$ The result can be checked with the first-order condition of the strategic informed trader's maximization problem at $t=n<N:\left[1-(1-\mu) \lambda_{n} a_{n}\right]\left[\gamma_{n}-2\left(1-\gamma_{n}\right) \mu \lambda_{n} H_{n}\right]=2 \mu \lambda_{n}\left[\gamma_{n}-\left(1-\gamma_{n}\right) \mu \lambda_{n} H_{n}\right] \alpha_{n}$. If $\gamma_{n}=0$, the equation is satisfied if $\lambda_{n}=0$ or if $\left[1-(1-\mu) \lambda_{n} a_{n}\right]=\mu \lambda_{n} \alpha_{n}$. It can be shown that the latter equation has no real solution. Therefore, $A_{n}=0$. 
that $\alpha_{n}<0$ for $\gamma_{n}$ low. It can be shown that if $\gamma_{1}$ is small enough, then at a linear equilibrium necessarily $\alpha_{1}<0$, and therefore there is manipulation. ${ }^{27}$ An important consequence, consistent with the simulations reported below, is that in the central case $\left(\gamma_{n}=\gamma^{N-n}\right)$ for $N$ large, we will have market manipulation, $\alpha_{n}<0$, in the first periods of the tâtonnement.

At stage $n<N$, if $0<\gamma_{n}<1$, the strategic informed trader faces the following tradeoff. If trade were consummated, the optimal action would be to trade as in the static model, choosing $\alpha_{n}$ positive and relatively high. If the market were not to open, the optimal response would be to choose a trading intensity $\alpha_{n}$ so as to make the price $p_{n}$ uninformative $\left(A_{n}=0\right.$ and $\left.p_{n}=p_{n-1}\right)$. He must balance reducing the informativeness of prices by choosing a low (and possibly negative) trading intensity $\alpha_{n}$ and trading intensely (choosing $\alpha_{n}$ close to the static equilibrium value) to obtain a high profit if trades are executed. If $\gamma_{n}=1$, the strategic informed trader behaves as in the static version of the model so that his response to private information is positive.

At any stage, the strategic informed trader's responsiveness to private information should be increasing in the probability of trading at that stage. If the strategic informed trader manipulates the market $\left(\alpha_{n}<0\right)$ and trade is realized, he will have an expected loss. But if there is no trading, the strategic informed trader's future expected profit will be higher, since he will preserve a greater informational advantage over other market participants. At stage $n$, the strategic informed trader's incentives to manipulate the market should be decreasing in the probability of trading $\gamma_{n}$, or equivalently, $\alpha_{n}$ increasing in $\gamma_{n}$. By the same token, and since $\gamma_{n}$ is increasing in $n, \alpha_{n}$ should be increasing in $n$.

We now present a simulation analysis of the main properties of the equilibrium in the general model. ${ }^{28}$ We have explored the behavior of the model with $\gamma_{n}=\gamma^{N-n}$ in the following parameter grid: $\rho$ in $\{1,2,4\}, \tau_{u}, \tau_{v}$, and $\tau_{\varepsilon}$ in $\{.5,1,2\}, \gamma$ in $\{.2, .3, .5, .6, .7, .8\}, \mu$ in $\{.2, .5, .8\}$ with $N$ up to 30 rounds. As stated in Section 3, for a moderate-horizon $N$ it is reasonable to choose $\gamma$ not too high so that the probability of trade is low at the beginning. We obtain the following results.

Summary of simulation results. Let $1>\mu>0$ and $\gamma_{n}=\gamma^{N-n}$. Then the simulations performed show that $\alpha_{n}$ is increasing in $n$ and in $\gamma, \tau_{n}$ is strictly convex in $n$, and, provided $N$ is large enough,

(i) there is always market manipulation, $\alpha_{n}<0$, in the first periods of the tâtonnement;

(ii) the informativeness of prices is very low during the first stages and increases fast as $n$ gets close to $N$;

(iii) the conditional volatility of $\operatorname{prices} \operatorname{var}\left(p_{n} \mid p_{n-1}\right)$ may be hump-shaped or increasing in $n$ and the responsiveness to information of the competitive agents $a_{n} \mathrm{U}$-shaped or decreasing; and

(iv) the total expected trading volume is U-shaped.

It is easy to generate speeds of learning close to those estimated by Biais, Hillion, and Spatt (1999). These authors estimate, in the period in which prices are informative, an order of magnitude for the precision of prices $\tau_{n}$ of $n^{3}$ instead of the order $n$ obtained by Vives (1995) for the competitive model. In our model, if we fit a curve of the type $K n^{k}$ to $\tau_{n}-\tau_{v}$ we easily find values for $k$ close to 3 for a range of periods in which $\tau_{n}$ is significantly different from $\tau_{v}$. For example, with $\rho=\tau_{\varepsilon}=\tau_{v}=\tau_{u}=1, \mu=.5$, and $N=10$, considering only periods for which $\tau_{n}>1.05 \tau_{v}$ we obtain that for $\gamma=.4$ (using the last four periods), $k=2.8$; for $\gamma=.5$ (using the last six periods), $k=2.7$; for $\gamma=.6$ (using the last nine periods), $k=2.6$.

It is worth noting that the expected length of the tâtonnement, for a fixed $N$, is decreasing in $\gamma$. As $\gamma$ tends to one, expected length tends to one, and as $\gamma$ tends to zero, expected length tends to $N$. A low $\gamma$ has the benefit of a "long" tâtonnement but at the cost of introducing more price manipulation. A high $\gamma$ means a "short" tâtonnement with less or no price manipulation. If the objetive is to maximize the expected informativeness of prices, an interior $\gamma$ will be optimal.

${ }^{27}$ For the case $N=2$ at the unique linear equilibrium for $\gamma_{1}$ close to zero, $\alpha_{1}<0$ and $\alpha_{1}$ is increasing in $\gamma_{1}$.

${ }^{28} \mathrm{We}$ do so given the difficulty in establishing analytically general properties of the equilibrium. Our iterative procedure to compute the equilibrium is available upon request. 
FIGURE 4

STRATEGIC INFORMED TRADER'S TRADING INTENSIVITY $\alpha_{n}$

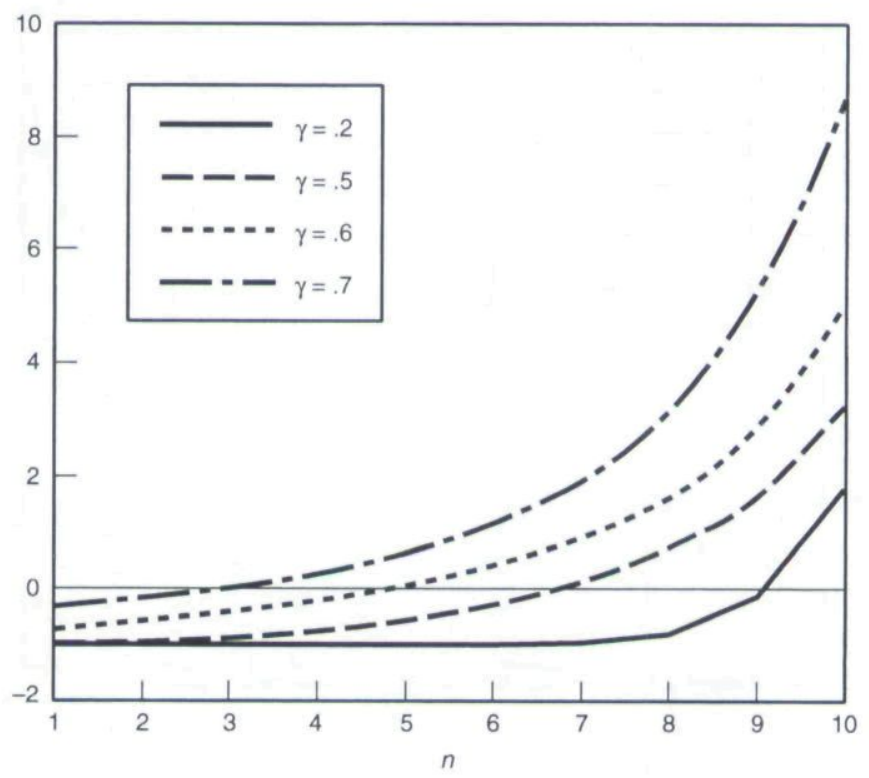

To understand the trading dynamics of the competitive informed agents, it is important to understand the dynamic behavior of the conditional volatility $\operatorname{var}\left(p_{n} \mid p_{n-1}\right)$. Information revelation by prices accelerates (decelerates) as $n$ increases when $\operatorname{var}\left(v \mid p_{n}\right)$ is concave (convex) in $n$. The following fact relates the two conditional variances.

Fact. The conditional volatility of prices $\operatorname{var}\left(p_{n} \mid p_{n-1}\right)$ is increasing (decreasing) in $n$ if and only if $\operatorname{var}\left(v \mid p_{n}\right)$ is concave (convex) in $n$.

Proof. We know that $\operatorname{var}\left(p_{n} \mid p_{n-1}\right)=\left(\tau_{n-1}\right)^{-1}-\left(\tau_{n}\right)^{-1}$ and $\tau_{n}=\left(\operatorname{var}\left(v \mid p_{n}\right)\right)^{-1}$. We have then that $\operatorname{var}\left(p_{n+1} \mid p_{n}\right) \geq \operatorname{var}\left(p_{n} \mid p_{n-1}\right)$ if and only if $\operatorname{var}\left(v \mid p_{n}\right)-\operatorname{var}\left(v \mid p_{n+1}\right) \geq \operatorname{var}(v \mid$ $\left.p_{n-1}\right)-\operatorname{var}\left(v \mid p_{n}\right)$. Rearranging terms, the inequality is equivalent to $\operatorname{var}\left(v \mid p_{n}\right)-\operatorname{var}(v \mid$ $\left.p_{n-1}\right) \geq \operatorname{var}\left(v \mid p_{n+1}\right)-\operatorname{var}\left(v \mid p_{n}\right)$, and the result follows. Q.E.D.

This means that the conditional volatility of prices is increasing (decreasing) if and only if information revelation accelerates (decelerates) as $n$ increases. Note also that if $\operatorname{var}\left(v \mid p_{n}\right)$ is concave in $n$, then $\tau_{n}=\left(\operatorname{var}\left(v \mid p_{n}\right)\right)^{-1}$ is convex in $n$.

In the examples that follow, illustrated by the figures and parametrized by $\gamma$ ranging from .2 to .7 , it is assumed that $N=10, \rho=\tau_{\nu}=\tau_{\bar{\omega}}=\tau_{\varepsilon}=1$, and $\mu=.5$. We comment on the results of the simulations for $\mu \leq .6$.

The strategic informed trader manipulates the market at the beginning $\left(\alpha_{n}<0\right.$ for $n$ low (Figure 4) except if $\gamma=.7$ ). As a result, the informativeness of prices is very low during the first stages and increases quite fast as $n$ gets close to $N$ (Figure 5). The conditional volatility of prices $\operatorname{var}\left(p_{n} \mid p_{n-1}\right)$ may be hump-shaped or increasing in $n$ (Figure 6), implying that the responsiveness to information of the competitive agents $a_{n}$ is $\mathrm{U}$-shaped or decreasing, respectively (Figure 7). The last situation happens when $\gamma$ is low $(\gamma=.2)$. Then information revelation accelerates as the tâtonnement progresses $\left(\operatorname{var}\left(v \mid p_{n}\right)\right.$ is concave in $\left.n\right)$. Otherwise, for larger $\gamma$ 's, $\operatorname{var}\left(v \mid p_{n}\right)$ is first concave and then convex in $n$, implying that $\operatorname{var}\left(p_{n} \mid p_{n-1}\right)$ is first increasing and then decreasing in $n$ (Figure 8). The total expected trading volume is U-shaped (Figure 9), and the result is driven by the fact that the strategic informed trader's expected trading volume is U-shaped (Figure 10). The explanation is as follows. The expected volume traded by informed traders (ignoring the volume traded among competitive informed agents) equals $\mu E\left|y_{n}\right|+E\left|x_{n}\right|=\left(\mu\left|\alpha_{n}\right|+(1-\mu) a_{n}\right)\left(\operatorname{var}\left(v \mid p_{n}\right)\right)^{1 / 2}$. For $\gamma$ not too high, this volume will have 
FIGURE 5

INFORMATIVENESS OF PRICES $\tau_{n}$

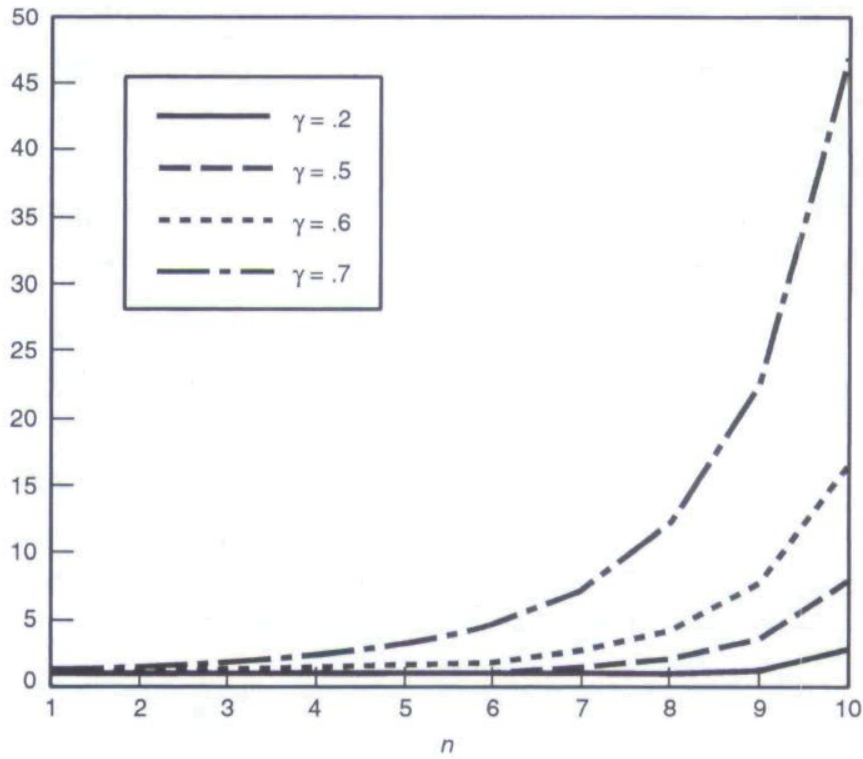

a U-shaped temporal pattern. The reason is that $\left|\alpha_{n}\right|$ has a U-shaped temporal pattern, and the same is true for $a_{n}$ except when $\gamma$ is low, in which case the evolution of $\left|\alpha_{n}\right|$ dominates and $\mu\left|\alpha_{n}\right|+(1-\mu) a_{n}$ also has a U-shaped temporal pattern. This in turn dominates the decreasing tendency of $\operatorname{var}\left(v \mid p_{n}\right)$.

A more detailed illustration of the simulations follows.

Responsiveness to private information. In Figure 4 we observe that $\alpha_{n}$ is increasing in $\gamma$ and in $n$. The dynamic behavior of $a_{n}$ depends on $\operatorname{var}\left(p_{n} \mid p_{n-1}\right)$. In the purely competitive model

\section{FIGURE 6}

CONDITIONAL VOLATILITY $\operatorname{var}\left(p_{n} \mid p_{n-1}\right)$

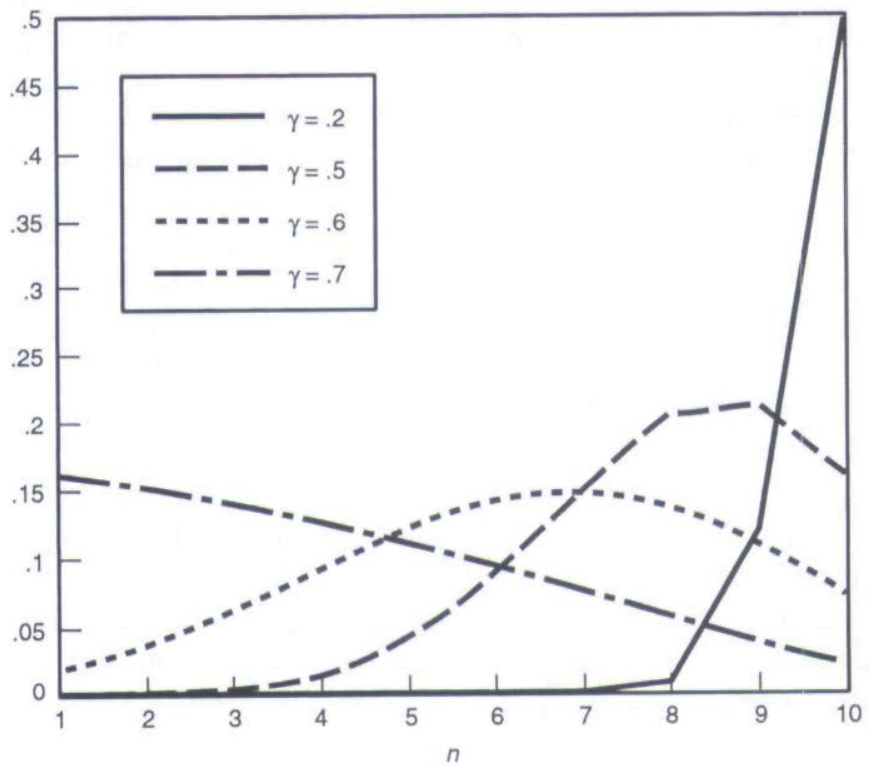


FIGURE 7

COMPETITIVE AGENT'S TRADING INTENSITY $a_{n}$

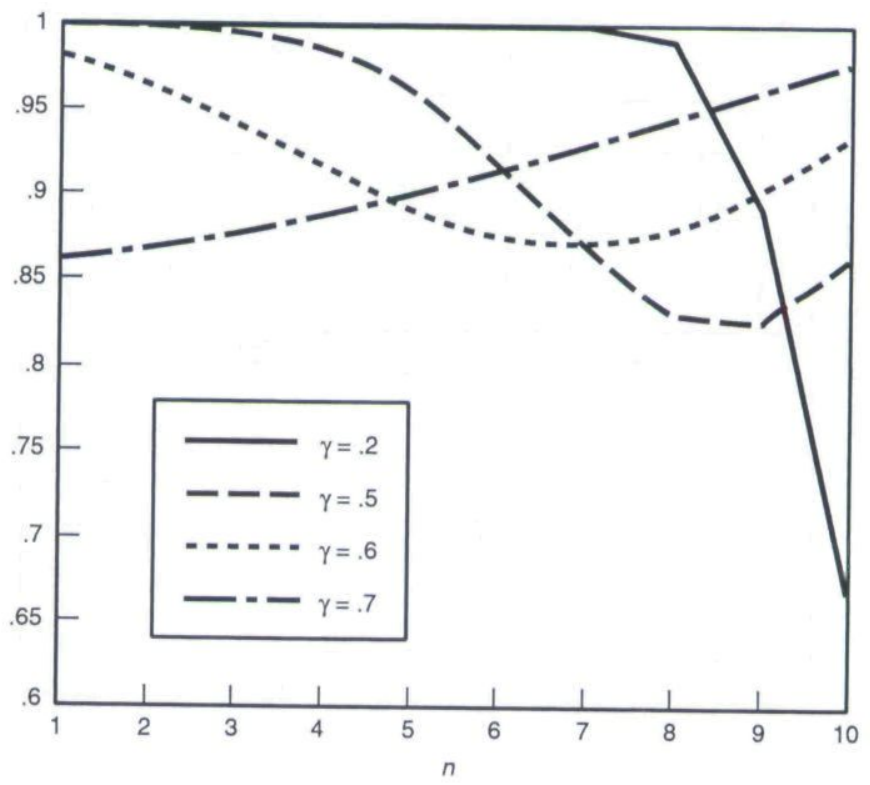

$(\mu=0), a_{n}$ increases monotonically to $a_{n}=\tau_{\varepsilon} / \rho$. In the presence of the strategic informed trader, $a_{n}$ may be monotonically increasing, monotonically decreasing, or U-shaped. If $\gamma$ is sufficiently low (see Figure $7, \gamma=.2$ ), the strategic informed trader will have very strong incentives to manipulate the market. As a result, prices will purvey almost no information during the first stages, and for $n$ close to $N$, information revelation will accelerate $\left(\operatorname{var}\left(v \mid p_{n}\right)\right.$ ) concave in $n$ (see Figure 8). This implies that $\operatorname{var}\left(p_{n} \mid p_{n-1}\right)$ is monotonically increasing (see Figure 6), and as a direct consequence, $a_{n}$ is also monotonically decreasing. On the contrary, if $\gamma$ is sufficiently high (see Figure $4, \gamma=.7$ ), the strategic informed trader will not manipulate the market, and informa-

FIGURE 8

RESIDUAL VARIANCE $\operatorname{var}\left(v \mid p_{n}\right)$

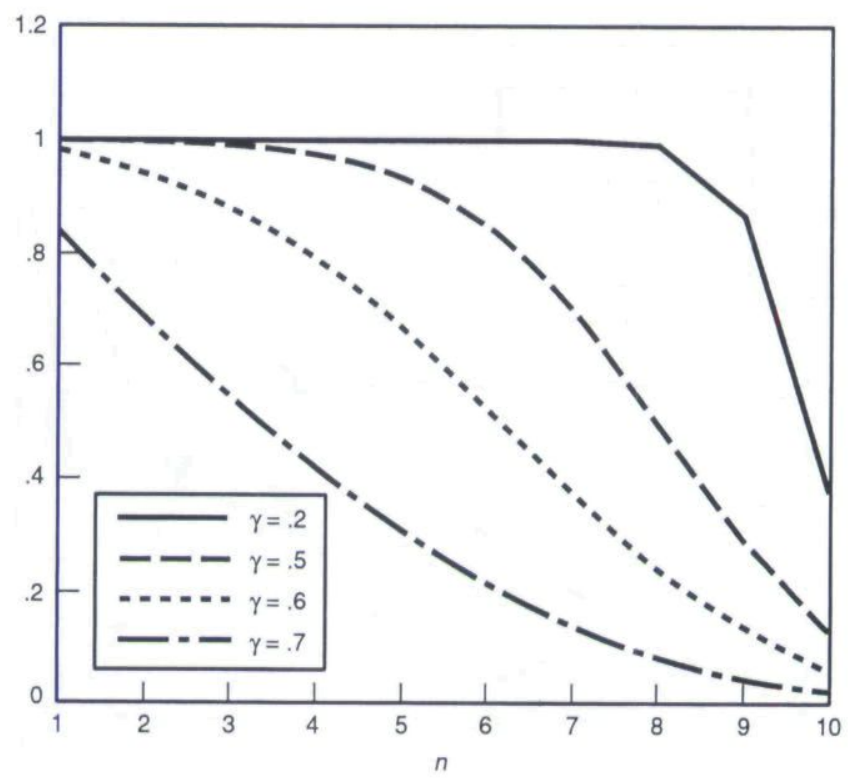


FIGURE 9

TOTAL EXPECTED TRADING VOLUME

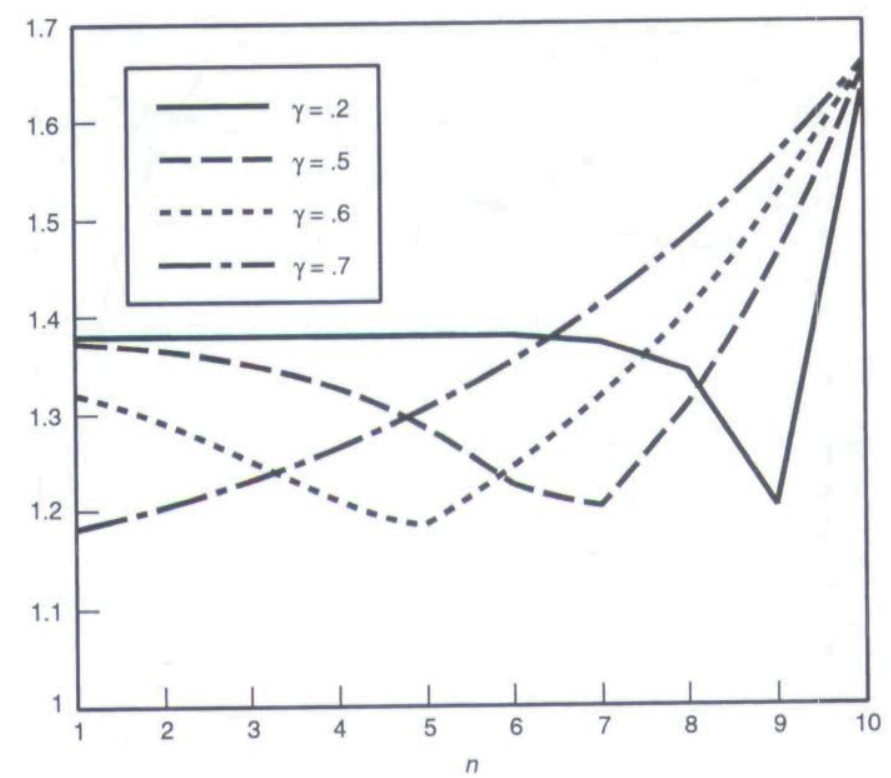

tion revelation will be fast from $n=1$ and will decelerate with $n\left(\operatorname{var}\left(v \mid p_{n}\right)\right.$ ) convex in $n$ (see Figure 8 ). Therefore, the conditional volatility of prices will be monotonically decreasing and $a_{n}$ will be monotonically increasing. For intermediate values of $\gamma\left(\gamma=.6\right.$, for example), $\operatorname{var}\left(v \mid p_{n}\right)$ is first concave and then convex in $n$. Then, $a_{n}$ is U-shaped.

Informativeness of prices. The price precision $\tau_{n}$ is monotonically increasing and convex in $n$ (see Figure 5). The informational efficiency of the process is increasing in $\gamma$. If $\gamma$ is very low (see Figure 5, $\gamma=.2$ ), the strategic informed trader will have very strong incentives to manipulate the market, and prices will purvey almost no information until $n$ gets very close to $N$. If $\gamma$ is suf-

\section{FIGURE 10}

STRATEGIC INFORMED TRADER'S TRADING VOLUME

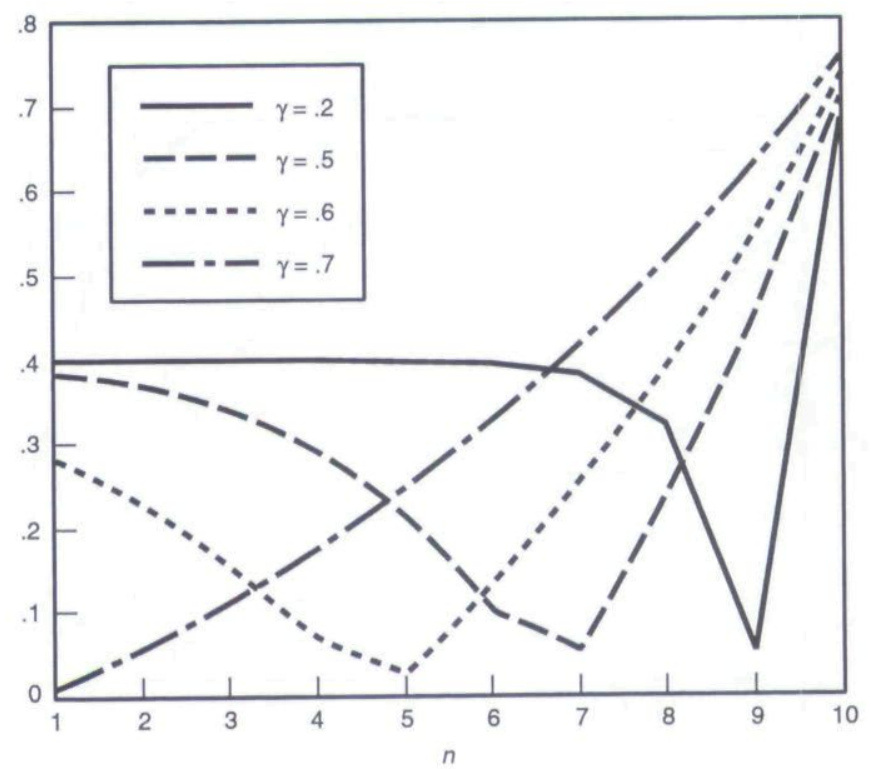




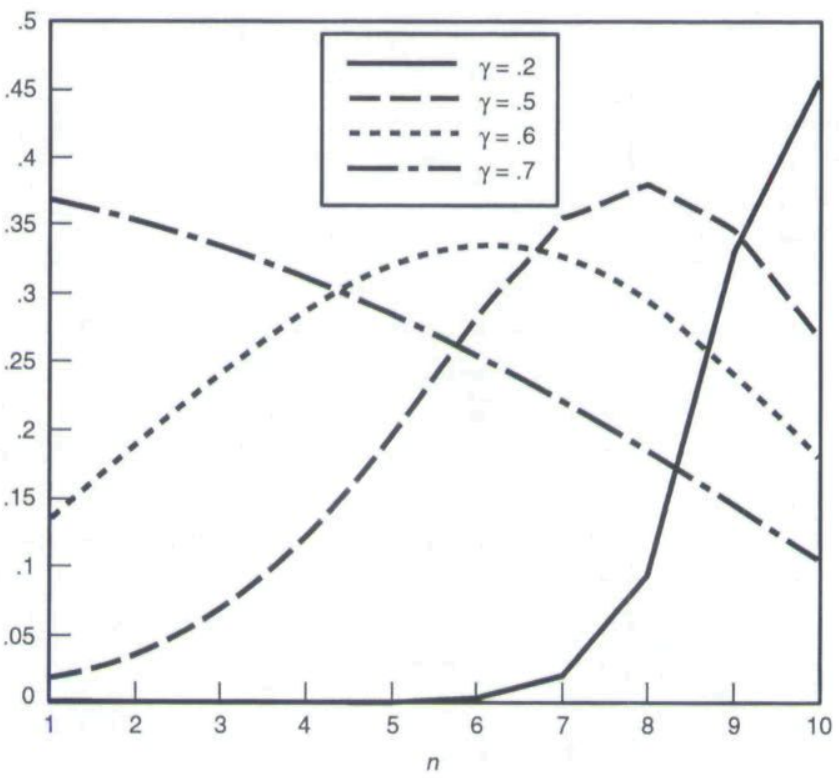

ficiently high relative to $N$ (see Figure $5, \gamma=.7$ ), the strategic informed trader will not manipulate the market, and information revelation will be relatively fast.

Market depth. In the purely competitive model $(\mu=0)$ the depth of the market tends monotonically to infinity at a rate of $n$, while in the monopolistic version of the model the depth of the market is generally U-shaped. In the general model, several patterns are possible. The depth of the market tends to be monotonically decreasing if $\gamma$ is low and monotonically increasing if $\gamma$ is high (see Figure 11, where $\lambda_{n}$ is displayed), having a U-shaped pattern for intermediate values. ${ }^{29}$

Trading volume. The dynamics of trading volume display a variety of patterns. In the purely competitive model $(\mu=0)$, trading volume is decreasing (at least for $n$ close to $N$ ), while in the purely monopolistic version of the model, trading volume is increasing. In the general version of the model, with $0<\mu<1$, expected trading volume may be increasing, decreasing, or U-shaped.

The strategic informed trader's expected trading volume, $\mu E\left|\lambda_{n}\right|=\mu(2 / \pi)^{1 / 2}\left(\left(\alpha_{n}\right)^{2} /\right.$ $\left.\tau_{n-1}\right)^{1 / 2}$, may be U-shaped or increasing depending on whether there is market manipulation or not (see Figure 10). If $\gamma$ is high, the strategic informed trader will choose $\alpha_{n}>0$ for all $n$. As $n$ increases, $\alpha_{n}$ will go up and, as in the purely monopolistic version of the model, the strategic informed trader's expected trading volume will increase. On the contrary, if $\gamma$ is low, the strategic informed trader will choose $\alpha_{1}<0$. As $n$ and $\gamma_{n}$ increase, the incentives to manipulate the market decrease and, as a consequence, $\alpha_{n}$ increases. But this means that, since $\alpha_{1}<0,\left(\alpha_{n}\right)^{2}$ decreases as $\alpha_{n}$ gets close to zero. Therefore, for $n$ low, the strategic informed trader's expected trading volume is decreasing since $\left(\alpha_{n}\right)^{2}$ is decreasing and the informativeness of past prices is increasing. As $n$ gets close to $N$, the strategic informed trader chooses a positive and increasing

${ }^{29}$ If $\gamma$ is low, market manipulation is extreme during the first stages, the order flow is almost equal to noise traders' demand, and market depth is very high. As $n$ increases, market manipulation decreases, the likelihood that the order flow reflects informed trading increases, and market depth decreases. However, for intermediate values of $\gamma$ and $n$ close to $N$, sufficient information may have been revealed for market depth to increase with $n$. If $\gamma$ is high, there is no manipulation, the order flow strongly reflects informed trading, and market depth is low at $n=1$. Information revelation is relatively fast (and decelerates with $n$ ). As $n$ grows, the information effect dominates and, as in the competitive model, market depth increases. 
$\alpha_{n}$, and then his expected trading volume becomes increasing. In general, the strategic informed trader's expected trading volume is decreasing (increasing) if and only if the strategic informed trader's trading intensity is negative (positive).

The expected volume traded by competitive informed agents, $E\left|y_{i n}\right|=(2 / \pi)^{1 / 2} a_{n}\left(1 / \tau_{\varepsilon}+\right.$ $\left.1 / \tau_{n-1}\right)^{1 / 2}$, is monotonically decreasing. The result should hold a fortiori in relation to the competitive model, since now there are cases in which $a_{n}$ decreases with $n$. On the other hand, if $\gamma$ increases, the expected volume traded by competitive informed agents tends to be smaller, since prices become more informative sooner.

The expected volume traded by market makers, $\left.E\left|\omega_{n}\right|=(2 / \pi)^{1 / 2}\left(\left(\sigma_{u}\right)^{2}+\left(A_{n}\right)^{2} / \tau_{n-1}\right)\right)^{1 / 2}$ increases with $\sigma_{u}$ and $A_{n}$, and decreases with $\tau_{n-1}$. Expected volume traded by market makers will be monotonically increasing in $n$ since $\tau_{n-1}$ increases more slowly than the average response to private information $A_{n}$ (because of the strategic behavior by the strategic informed trader) ${ }^{30}$

The expected total trading volume, ETV $V_{n}=(1 / 2 \pi)^{1 / 2}\left\{(1-\mu) a_{n}\left(1 / \tau_{\varepsilon}+1 / \tau_{n-1}\right)^{1 / 2}+\right.$ $\left.\left.\mu\left(\left(\alpha_{n}\right)^{2} / \tau_{n-1}\right)^{1 / 2}+\left(\left(\sigma_{u}\right)^{2}+\left(A_{n}\right)^{2} / \tau_{n-1}\right)\right)^{1 / 2}+\sigma_{u}\right\}$, tends to be U-shaped if $\gamma$ is low, as the strategic informed trader's expected trading volume is U-shaped. On the other hand, if $\gamma$ is high, the expected trading volume coming from the strategic informed trader increases fast and the expected trading volume coming from competitive informed agents decreases relatively slowly. In this case, expected total trading volume will tend to be monotonically increasing. ET $V_{n}$ is driven by the trades of the strategic informed trader (see Figure 9).

We shall now briefly analyze the comparative dynamics of the equilibrium with respect to the size of the strategic informed trader $\mu$ and the horizon $N$.

Comparative dynamics with respect to $\mu$. If $1>\mu^{\prime}>\mu>0$, then the simulations ${ }^{31}$ show that $\tau_{n}^{\prime}<\tau_{n}$, var $p_{n}^{\prime}<\operatorname{var} p_{n}$, and $E\left[T V_{n}^{\prime}\right]<E\left[T V_{n}\right]$. In general, we also have that $\left|\alpha_{n}^{\prime}\right|<\left|\alpha_{n}\right|$ with the possible reversal of the inequality when the $\alpha$ 's are negative and close to zero. The effect of $\mu$ on $a_{n}$ and $\lambda_{n}$ is ambiguous. Furthermore, with the possible exception of short horizons, the expected utility of a single informed competitive agent increases in the presence of the strategic informed trader and in his size $\mu$. The expected profit of the strategic informed trader also increases with $\mu$.

That is, in the economy in which the strategic informed trader is smaller (and the competitive sector larger), the strategic informed trader responds more to his private information, prices reveal more information and are more volatile, and there is more trading. If $\mu^{\prime}>\mu$, the effect of the strategic informed trader's demand on the price and on the informativeness of the price is higher, and he becomes more cautious. The expected trading volume is higher in the economy with a larger competitive sector. Indeed, the expected volume traded by the competitive sector is higher simply because this sector is larger while the trade per agent is more or less the same. Furthermore, market makers trade more as well because they face a less severe adverse selection problem (except perhaps at the end of the horizon). Finally, the expected volume traded by the strategic informed trader, at least in the first periods, is higher since the lower the impact his demand has on prices, the higher his willingness to trade. ${ }^{32}$

The previous comparative dynamic results have interesting implications for the relative welfare of the strategic informed trader and the competitive informed agents. The expected profits of the strategic informed trader in period $n$, conditional on there being trade in this period, are

${ }^{30}$ If there is market manipulation, most of the trading coming from competitive informed agents is absorbed by the strategic informed trader. As $n$ increases, the incentives to manipulate the market decrease, and market makers absorb a higher share of trading coming from the competitive informed sector. At some stage, $\alpha_{n}>0$ and market makers become the counterpart for all informed and noise trading.

${ }^{31}$ We have simulated the model in the following range of parameter values: $\rho$ in $\{1,2,4\}, \tau_{u}, \tau_{v}$, and $\tau_{\varepsilon}$ in $\{.5,1,2\}, \gamma$ in $\{.3, .5, .7\}$, and $\mu$ in $\{0, .2, .5, .8,1\}$ with $N$ up to 30 rounds.

${ }^{32}$ The effect of an increase in $\mu$ on $a_{t}$ is not uniform (if $\mu^{\prime}>\mu$ there is a critical $n$ such that $a_{t}^{\prime}\left\langle a_{t}\right.$ for $t>n$ and $a_{t}^{\prime}>a_{t}$ for $\left.t \leq n\right)$. An increase in $\mu$ decreases $\tau_{n}$. If $\mu^{\prime}>\mu$, then there is a critical $n$ such that $\left(\lambda_{t}^{\prime}\right)^{-1}<\left(\lambda_{t}\right)^{-1}$ for $t>n$ and $\left(\lambda_{t}^{\prime}\right)^{-1}>\left(\lambda_{t}\right)^{-1}$ for $t \leq n$. Market depth increases with $\mu$ at the first stages of the process and decreases with $\mu$ when $n$ gets close to $N$.

(c) RAND 2001. 
given by $\mu \alpha_{n} / \tau_{n}$. The expected utility of a competitive informed agent, conditional on there being trade in period $n$, is given by $-\left(1+\tau_{\varepsilon} / \tau_{n-1}\right)^{-1 / 2}$. Given that the presence of the strategic informed trader tends to make prices less informative (with the possible exception of short horizons), the competitive traders benefit (individually) from it. Furthermore, a larger strategic informed trader makes $\alpha_{n}$ smaller (when positive) and the price precision $\tau_{n}$ also smaller. The simulations show that the expected profit, $\mu \alpha_{n} / \tau_{n}$, in general increases with $\mu$ (although $\alpha_{n} / \tau_{n}$ may decrease with $\mu),{ }^{33}$ and therefore the strategic informed trader prefers that the competitive sector be small. We also find that the ex ante expected profit of the strategic informed trader (as of period 0 ) is increasing in $\mu$.

In summary, an informed competitive trader prefers to have a large informed trader around because then prices are less informative and the trader can profit from it, while the strategic informed trader prefers a small competitive sector. The strategic informed trader is creating a positive externality for the competitive informed agents.

Comparative dynamics with respect to $N$. In the purely competitive economy $(\mu=0)$, increasing $N$ increases the informativeness of prices at the rate of $N$ and decreases the volume of trading. In the purely strategic version of the model $(\mu=1)$, for any given $\gamma$ there is an upper bound for the price precision $\bar{\tau}$, no matter the length $N$ of the horizon. Our simulations support the conjecture that this is also the case when $\mu>0 .{ }^{34}$

For example, with $\mu=.5, \rho=\tau_{\varepsilon}=\tau_{v}=\tau_{u}=1$, and $\gamma=.5$ as fixed exogenous parameters, we see that $a_{N}, \alpha_{N}, \tau_{N}, \lambda_{N}, \operatorname{var}\left(p_{N} \mid p_{N-1}\right), \operatorname{var}\left(p_{N}\right)$, and the expected volume traded by the different types of agents all change by less than $.001 \%$ when $N$ increases from 10 to 20 . A larger $\mu$ implies a lower limit value for the price precision, and this limit is attained in fewer rounds of trade. Let $\rho=\tau_{\varepsilon}=\tau_{v}=\tau_{u}=1$, and $\gamma=.3$. Then for $\mu=.5, \bar{\tau}=3.416$ and the bound is attained in about 10 rounds (up to 9 decimals); for $\mu=.1, \bar{\tau}=5.416$ and the bound is attained in close to 11 rounds; for $\mu=.01, \bar{\tau}=6.166$ and the bound is attained in less than 15 rounds. In the first case we get within less than $1 \%$ of the upper bound at $N=3$; in the second, at $N=4$; and in the third, at $N=5$. Indeed, when $\mu$ increases, the average responsiveness to information, $A_{n}$, tends to decrease, and this affects the informativeness of prices.

Robustness. It is possible to show that the general pattern of results obtained also hold in the case that the strategic and the competitive informed agents use demand schedules instead of market orders: In the presence of the strategic informed trader there is market manipulation, price precision is bounded above, and volume is $\mathrm{U}$-shaped.

\section{Concluding remarks}

- In this article we have provided a model of an information tâtonnement inspired in the preopening auction of some continuous trading systems. The interaction between a strategic informed trader and a sector of competitive informed agents yields outcomes consistent with the empirical evidence available from the Paris Bourse (Biais, Hillion, and Spatt, 1999). Indeed, we see how the presence of the strategic informed trader slows down at first and later accelerates the transmission of information by prices. The price precision tends to increase sharply toward the end of the tâtonnement. However, the price does not fully reveal the fundamental value of the asset, no matter how many rounds the tâtonnement has. Furthermore, trading volume displays a U-shaped pattern driven by the strategic informed trader's activity. All these robust findings in our model are consistent with the empirical evidence.

${ }^{33}$ The conditional expected profit $\mu \alpha_{n} / \tau_{n}$ may be nonmonotone in $\mu$ for intermediate values of $n$ when $\alpha$ is close to zero.

${ }^{34}$ Simulations have been performed in the following range: $\rho$ in $\{1,2,4\}, \tau_{u}, \tau_{v}$, and $\tau_{\varepsilon}$ in $\{.5,1,5\}, \mu$ between .01 and 1 with a step of .05 , and $\gamma$ with the same step from .01 until .5. The upper bound for $\tau$ is attained in 30 rounds or less. For $\gamma$ 's up to .7 and $\mu$ 's no smaller than .2, the upper bound for $\tau$ is attained in 40 rounds or less. 
At the same time, our model has characterized precisely the market-manipulation strategy of the strategic informed trader in terms of contrarian behavior. Indeed, the strategic informed trader attempts to manipulate the market at the beginning of the process by taking an offsetting position to the competitive informed traders. The aim of such a contrarian strategy is to keep the informativeness of prices low. Therefore, at the beginning there is quite a bit of contrarian activity that subsequently subsides to active trading in the direction of the information of the strategic informed trader. The presence of the strategic informed trader creates a public good for the competitive informed traders: A low informativeness of the price and opportunities to profit from it. On the other side of the coin, the strategic informed trader would like to keep the competitive informed sector as small as possible.

In summary, the presence of strategic behavior in a context where there are also competitive informed agents yields a temporal pattern of evolution of basic market parameters consistent with the evidence available in the pretrade period in the Paris Bourse. Furthermore, we uncover and characterize the use of a contrarian strategy by the strategic informed trader to manipulate the market. At the same time, the model makes clear that introducing a random opening time, like in Xetra, limits but does not eliminate the incentives to manipulate the market. Market manipulation only arises in those periods in which the probability that the market opens is relatively low.

Among the direct extensions of the model that could be explored, we could look at what happens if the strategic informed trader is uncertain about the risk aversion or the precision of information of the competitive traders: what if there is more than one strategic informed trader, or what if the strategic informed trader is risk averse? With respect to the latter issue, the results of Holden and Subrahmanyam $(1992,1994)$ seem to indicate that in such a case, information should be incorporated into prices much more quickly. Another relevant extension would inquire about the incentives to form coalitions of competitive informed traders. The benefit of forming a coalition is to share and improve information about the liquidation value of the risky asset. The cost is the internalization of information leakages and the subsequent incentive to refrain from trading aggressively. Still another extension would consider introducing the costs of submitting orders.

On another front, our model points toward market design to facilitate price discovery and prevent manipulation. For example, can activity rules for traders be devised to improve the performance of the tâtonnement? This alludes to the more general issue of finding a market design that performs well and is robust to changes in the environment. The topic is of relevance for a range of market environments in which agents can revise their trades before the market opens, including electricity auctions and parimutuel betting as well as the preopening period in stock markets.

\section{Appendix}

- In this Appendix we provide proofs of Proposition 1, Lemma 1, and Lemma A1 (with statement), and we develop the analysis of the equilibrium in demand schedules (Proposition A1).

Proof of Proposition 1. At a linear equilibrium, and given our assumptions, all random variables are normally distributed. Maximization of a CARA utility function by competitive agent $i$ then yields at stage $n$

$$
X_{i n}\left(s_{i}, p^{n-1}\right)=E\left\{\left(v-p_{n}\right) \mid s_{i}, p^{n-1}\right\} /\left[\rho \operatorname{var}\left\{\left(v-p_{n}\right) \mid s_{i}, p^{n-1}\right\}\right],
$$

where $p_{n}=E\left(v \mid \omega^{n}\right)$ from the competition among market makers, and where $\omega_{n}$ is the $n$-period order flow, which is given by $\omega_{n}=\mu y_{n}+x_{n}+u_{n}$, with $x_{n}=\int_{\mu}^{1} X_{i n}\left(s_{i}, p^{n-1}\right) d i$. Equilibria will be symmetric in the treatment of the competitive informed agents. Let the candidate equilibrium strategy $X_{i n}\left(s_{i}, p^{n-1}\right)=a_{n} s_{i}+\phi_{n}\left(p^{n-1}\right)$, where $\alpha_{n}>0$, and $\phi_{n}(\cdot)$ is a linear function. Let the strategic informed trader's candidate equilibrium strategy be $Y_{n}\left(v, p^{n-1}\right)=a_{n} v+\Phi_{n}\left(p^{n-1}\right)$, where $\Phi_{n}(\cdot)$ is also a linear function. The corresponding order flow will be given by $\omega_{n}=\left[\mu \alpha_{n}+(1-\mu) a_{n}\right] v+\mu \Phi_{n}\left(p^{n-1}\right)+$ $(1-\mu) \phi_{n}\left({ }^{n-1}\right)+u_{n}$, since $x_{n}=\int_{\mu}^{1} X_{i n}\left(s_{i}, p^{n-1}\right) d i=\int_{\mu}^{1}\left\{a_{n} s_{i}+\phi_{n}\left(p^{n-1}\right)\right\} d i=(1-\mu) a_{n} v+(1-\mu) \phi_{n}\left(p^{n-1}\right)$, using our convention about the average signal of the competitive agents. Note that the informational content of the order flow is summarized in the variable $z_{n}=A_{n} v+u_{n}$, where $A_{n}=\mu \alpha_{n}+(1-\mu) a_{n}$. Similarly to Vives (1995), (1) RAND 2001. 
we obtain that the optimal strategy of the competitive informed $i$ agent is given by $X_{i n}\left(s_{i}, p^{n-1}\right)=a_{n}\left(v-p_{n-1}\right)$ with $a_{n}=\left[\rho\left(1 / \tau_{\varepsilon}+\operatorname{var}\left\{p_{n} \mid p_{n-1}\right\}\right)\right]^{-1}=\left\{\rho\left[\left(\tau_{\varepsilon}\right)^{-1}+\left(\tau_{n-1}\right)^{-1}-\left(\tau_{n}\right)^{-1}\right]\right\}^{-1}$.

Consider now the problem of the strategic informed trader. At stage $n$ the strategic informed trader will face the following expected profit conditional on his information:

$$
E\left\{\pi_{n} \mid v, p^{n-1}\right\}=\gamma_{n} E\left\{\left(v-p_{n}\right) \mu y_{n} \mid v, p^{n-1}\right\}+\left(1-\gamma_{n}\right) E\left\{\pi_{n+1} \mid v, p^{n-1}\right\}
$$

where $E\left\{\pi_{n+1} \mid v, p^{n-1}\right\}$ is the expected continuation profit. Suppose that

$$
E\left\{\pi_{n+1} \mid v, p^{n}\right\}=\mu\left(H_{n}\left(v-p_{n}\right)^{2}+\delta_{n}\right),
$$

where $H_{n}$ and $\delta_{n}$ are constants. Then the strategic informed trader's objective function at stage $n$ is given by

$$
E\left\{\pi_{n} \mid v, p^{n-1}\right\}=\gamma_{n} E\left\{\left(v-p_{n}\right) \mu y_{n} \mid v, p^{n-1}\right\}+\left(1-\gamma_{n}\right) \mu\left[H_{n} E\left\{\left(v-p_{n}\right)^{2} \mid v, p^{n-1}\right\}+\delta_{n}\right] .
$$

It can be checked that

$$
E\left\{p_{n} \mid v, p^{n-1}\right\}=p_{n-1}+\lambda_{n}(1-\mu) a_{n}\left(v-p_{n-1}\right)+\mu \lambda_{n} y_{n}
$$

and

$$
E\left\{\left(v-p_{n}\right)^{2} \mid v, p^{n-1}\right\}=\left[\left\{1-\lambda_{n}(1-\mu) a_{n}\right\}\left(v-p_{n-1}\right)-\mu \lambda_{n} y_{n}\right]^{2}+\left(\lambda_{n}\right)^{2}\left(1 / \tau_{u}\right),
$$

since $u_{n}$ is independent of $\left\{v, p^{n-1}\right\}$. By substituting these expressions into the objective function $E\left\{\pi_{n} \mid v, p^{n-1}\right\}$, we have

$$
\begin{aligned}
& \mu\left(\gamma_{n} y_{n}\left[\left\{1-\lambda(1-\mu) a_{n}\right\}\left(v-p_{n-1}\right)-\mu \lambda_{n} y_{n}\right]\right. \\
& \left.\quad+\left(1-\gamma_{n}\right) H_{n}\left[\left\{1-\lambda_{n}(1-\mu) a_{n}\right\}\left(v-p_{n-1}\right)-\mu \lambda_{n} y_{n}\right]^{2}+\left(1+\gamma_{n}\right) H_{n}\left(\lambda_{n}\right)^{2}\left(1 / \tau_{u}\right)+\left(1-\gamma_{n}\right) \delta_{n}\right) .
\end{aligned}
$$

The first-order condition directly yields $y_{n}=\alpha_{n}\left(v-p_{n-1}\right)$, with

$$
\alpha_{n}=\left\{\left[1-(1-\mu) \lambda_{n} a_{n}\right] /\left[2 \mu \lambda_{n}\right]\right\}\left\{\left[\gamma_{n}-2\left(1-\gamma_{n}\right) \mu \lambda_{n} H_{n}\right] /\left[\gamma_{n}-\left(1-\gamma_{n}\right) \mu \lambda_{n} H_{n}\right]\right\}
$$

The second-order condition is given by $\lambda_{n} \mu\left[\gamma_{n}-\left(1-\gamma_{n}\right) \mu \lambda_{n} H_{n}\right]>0$. By substituting (back) $y_{n}=\alpha_{n}\left(v-p_{n-1}\right)$ into the objective function, we directly obtain the equations

$$
\begin{aligned}
H_{n} & =\left(1-\lambda_{n+1} A_{n+1}\right)\left[\gamma_{n+1} \alpha_{n+1}+\left(1-\gamma_{n+1}\right) H_{n+1}\left(1-\lambda_{n+1} A_{n+1}\right)\right] \\
\delta_{n} & =\left(1-\gamma_{n+1}\right)\left[\delta_{n+1}+\left(\lambda_{n}\right)^{2} H_{n+1} / \tau_{u}\right] .
\end{aligned}
$$

Finally, the boundary conditions $H_{N}=0, \delta_{N}=0$, and $2 \mu \alpha_{N} \lambda_{N}=\left[1-(1-\mu) \lambda_{N} a_{N}\right]$ may easily be found by solving the strategic informed trader's problem at stage $N$. The objective function is given by $y_{N}\left[\left\{1-\lambda_{N}(1-\mu) a_{N}\right\}\left(v-p_{N-1}\right)-\right.$ $\mu \lambda_{N} y_{N}$ ], so that $H_{N}=0=\delta_{N}$. Moreover, it is obvious that no new profit may be made after trade is consummated at $t=N$, which implies that $H_{N}$ and $\delta_{N}$ must be equal to zero. The optimal strategy is $x_{N}=\alpha_{N}\left(v-p_{N-1}\right)$, with $\alpha_{N}=\left[1-(1-\mu) \lambda_{N} a_{N}\right] /\left(2 \mu \lambda_{N}\right)$.

Now we will check the inequalities in Corollary 1. From the first- and second-order conditions we know that there is a (linear) equilibrium if and only if there are $\alpha_{n}$ and $a_{n}$ for $n=1, \ldots, N$ that satisfy the equation system (for $n=1, \ldots, N$ )

$$
\begin{aligned}
& a_{n}=\left\{\rho\left[\left(\tau_{\varepsilon}\right)^{-1}+\left(\tau_{n-1}\right)^{-1}-\left(\tau_{n}\right)^{-1}\right]\right\}^{-1} \\
& \alpha_{n}=\left\{\left[1-(1-\mu) \lambda_{n} a_{n}\right] /\left[2 \mu \lambda_{n}\right]\right\}\left\{\left[\gamma_{n}-2\left(1-\gamma_{n}\right) \mu \lambda_{n} H_{n}\right] /\left[\gamma_{n}-\left(1-\gamma_{n}\right) \mu \lambda_{n} H_{n}\right]\right\}
\end{aligned}
$$

and the second-order conditions $\lambda_{n} \mu\left[\gamma_{n}-2\left(1-\gamma_{n}\right) \mu \lambda_{n} H_{n}\right]>0$, where $H_{n}=\left(1-\lambda_{n+1} A_{n+1}\right)\left[\gamma_{n+1} \alpha_{n+1}+(1-\right.$ $\left.\left.\gamma_{n+1}\right) H_{n+1}\left(1-\lambda_{n+1} A_{n+1}\right)\right], \lambda_{n}=\tau_{u} A_{n} / \tau_{n}, A_{n}=\mu \alpha_{n}+(1-\mu) a_{n}, H_{N}=0, \delta_{N}=0, \tau_{0}=\tau_{v}$, and $\tau_{n}=\tau_{n-1}+\tau_{u}\left(A_{n}\right)^{2}$, for $n=1, \ldots, N$. This system of equations in $\left\{a_{1}, \ldots, a_{N}, \alpha_{1}, \ldots, \alpha_{N}\right\}$ can be transformed into an equivalent system (1) RAND 2001 . 
in the unknowns $\left\{a_{1}, \ldots, a_{N}, \lambda_{1}, \ldots, \lambda_{N}\right\}$ :

$$
\left[\gamma_{n}-\left(1-\gamma_{n}\right) \mu \lambda_{n} H_{n}\right]\left\{\left(\tau_{n} / \tau_{u}\right)\left(\lambda_{n}\right)^{2}-(1-\mu) a_{n} \lambda_{n}\right\}-\left[1-(1-\mu) \lambda_{n} a_{n}\right]\left[\gamma_{n}-2\left(1-\gamma_{n}\right) \mu \lambda_{n} H_{n}\right] / 2=0 .
$$

From a simple inspection of the second-order conditions with $\mu>0, \lambda_{n} \mu\left[\gamma_{n}-\left(1-\gamma_{n}\right) \mu \lambda_{n} H_{n}\right]>0$, it is easy to see that in equilibrium $\lambda_{n}>0$, since $\mu, \gamma_{n},\left(1-\gamma_{n}\right)$, and $H_{n}$ are all nonnegative. ${ }^{35}$ Consider the equation in $a_{n}$, $a_{n}=\left\{\rho\left[\left(\tau_{\varepsilon}\right)^{-1}+\left(\tau_{n-1}\right)^{-1}-\left(\tau_{n}\right)^{-1}\right]\right\}^{-1}$. As in Vives (1995), given $\alpha_{n}$ or $\lambda_{n}$, it is easily checked that this is a cubic equation with a unique positive root. Now, given nonnegative $H_{n}, \tau_{n}$, and $a_{n},(* *)$ is a cubic polynomial equation in $\lambda_{n}$ of the form $F\left(\lambda_{n}\right)=0$. It can be checked that, given nonnegative $H_{n}, \tau_{n}$, and $a_{n}$, this equation has a negative solution (since $F(0)<0$ and, if $\lambda_{n}$ tends to $-\infty, F\left(\lambda_{n}\right)$ tends to $\left.+\infty\right)$, a solution in the open interval $\left(0, \gamma_{n} /\left[\left(1-\gamma_{n}\right) \mu H_{n}\right]\right)$, and a third solution strictly greater than $\gamma_{n} /\left[\left(1-\gamma_{n}\right) \mu H_{n}\right]$, since $F(0)<0, F\left(\lambda_{n}\right)>0$ if $\lambda_{n}=\gamma_{n} /\left[\left(1-\gamma_{n}\right) \mu H_{n}\right]$, and $F\left(\lambda_{n}\right)$ tends to $-\infty$ if $\lambda_{n}$ tends to $+\infty$. While neither the smallest nor the largest root satisfies the second-order condition $\lambda_{n} \mu\left[\gamma_{n}-\left(1-\gamma_{n}\right) \mu \lambda_{n} H_{n}\right]>0$, the middle root does satisfy it. In consequence, $0<\mu \lambda_{n} H_{n}<\gamma_{n} /\left(1-\gamma_{n}\right)$. The rest of the inequalities follow easily. Q.E.D.

Proof of Lemma 1. The results are immediate from the fact that if $z$ is normally distributed with $E z=0$, then $E|z|=$ $(2 / \pi)^{1 / 2}[\operatorname{var} z]^{1 / 2}$ and from the equilibrium expressions in Proposition 2. We know that $x_{n i}=a_{n}\left(s_{i}-p_{n-1}\right)$ and therefore $E x_{i n}=0\left(E s_{i}=E v=E p_{n}\right)$. Furthermore, $\operatorname{var} x_{i n}=\left(a_{n}\right)^{2} \operatorname{var}\left(s_{i}-p_{n-1}\right)$ and $\operatorname{var}\left(s_{i}-p_{n-1}\right)=1 / \tau_{\varepsilon}+\operatorname{var}\left(v-p_{n-1}\right)=$ $1 / \tau_{\varepsilon}+1 / \tau_{n-1}$ because $\operatorname{var}\left(v-p_{n-1}\right)=\operatorname{var}\left(v \mid p_{n-1}\right)=1 / \tau_{n-1} .{ }^{36}$ We have then that $E\left|x_{i n}\right|=(2 / \pi)^{1 / 2} a_{n}\left(1 / \tau_{\varepsilon}+\right.$ $\left.1 / \tau_{n-1}\right)^{1 / 2}$, given that $a_{n} \geq 0$, and, similarly, $E\left|y_{n}\right|=(2 / \pi)^{1 / 2}\left(\left(\alpha_{n}\right)^{2} / \tau_{n-1}\right)^{1 / 2}$. Finally, $E\left|\omega_{n}\right|=E \mid \int_{\mu}^{1} x_{i n} d i+\mu y_{n}+$ $u_{n}|+E| A_{n}\left(v-p_{n-1}\right)+u_{n} \mid$, where $x_{n}=\int x_{n i} d i=(1-\mu) a_{n}\left(v-p_{n-1}\right)$. Since $u_{n}$ is independent of $\left(v-p_{n-1}\right)$ and $E \omega_{n}=0$, we have that $E\left|\omega_{n}\right|=(2 / \pi)^{1 / 2}\left[\operatorname{var} \omega_{n}\right]^{1 / 2}=(2 / \pi)^{1 / 2}\left[\operatorname{var} u_{n}+\left(A_{n}\right)^{2} \operatorname{var}\left(v-p_{n-1}\right)\right]^{1 / 2}=(2 / \pi)^{1 / 2}\left[\left(\sigma_{u}\right)^{2}+\left(A_{n}\right)^{2} / \tau_{n-1}\right]^{1 / 2}$. The expression for $E T V_{n}$ follows. Q.E.D.

Lemma A1. Let $q_{n}=\lambda_{n} H_{n}$. The solution of the difference equation system $(1,2,3)$ in Proposition 3 is given by starting from $q_{N}=0$ and iterating backward for $q_{N-1}, \ldots, q_{1}$ using the unique root of the cubic equation $8\left(1-\gamma_{n-1}\right)\left(q_{n-1}\right)^{3}-$ $8 \gamma_{n-1}\left(q_{n-1}\right)^{2}-2\left(1-\gamma_{n-1}\right) k_{n} q_{n-1}+\gamma_{n-1} k_{n}=0$, where $k_{n}=\left(\gamma_{n}\right)^{3} /\left[\gamma_{n}-2 q_{n}\left(1-\gamma_{n}\right)\right]$. At stage $n$, the solution $q_{n}$ lies in the interval $\left(0, k_{n} / 2\right)$. Then iterate forward for each of the following variables in the order listed:

$$
\begin{aligned}
\tau_{n} & =2\left[1-q_{n}\left(1-\gamma_{n}\right) / \gamma_{n}\right] \tau_{n-1} \\
\alpha_{n} & =\left\{\tau_{n} /\left(2 \tau_{u}\right)\left[\gamma_{n}-2 q_{n}\left(1-\gamma_{n}\right)\right] /\left[\gamma_{n}-q_{n}\left(1-\gamma_{n}\right)\right]\right\}^{1 / 2} \\
\lambda_{n} & =\tau_{u} \alpha_{n} / \tau_{n},
\end{aligned}
$$

starting from $\tau_{0}=\tau_{v}$. Finally $H_{n}$ and $\delta_{n}$ are calculated using the equations (2) and (3) above.

Corollary Al. We have that $\tau_{n}=2^{n} \tau_{v} \prod_{t=1}^{n}\left[1-q_{t}\left(1-\gamma_{t}\right) / \gamma_{t}\right]$.

Proof. Available upon request. Similar to the methods developed in Holden and Subrahmanyam (1992) and also in Foster and Viswanathan (1993).

Equilibrium with demand schedules. Consider a version of the general model in which both the strategic and the competitive informed agents submit demand schedules instead of market orders. At stage $n$, informed agent $i$ submits a demand schedule $X_{n i}\left(p_{n} ; s_{i}, p^{n-1}\right)$, contingent on the private signal $s_{i}$ he has and the past history of prices. Similarly, the strategic informed trader's strategy at $n$ is a demand function contingent in his private information $(v)$ and past prices, $Y_{n}\left(p_{n} ; v, p^{n-1}\right)$. As before, noise traders are assumed to submit at round $n$ the order $u_{n}$, and market makers quote prices efficiently on the basis of public information and the aggregate limit order book, which is just a noisy version of the aggregate orders of informed agents, $L_{n}\left(p_{n}\right)=\mu y_{n}\left(p_{n}\right)+x_{n}\left(p_{n}\right)+u_{n}$, where $x_{n}\left(p_{n}\right)=\int_{\mu}^{1} x_{n i}\left(p_{n} ; s_{i}, p_{n-1}\right)$.

Proposition A1. There exists a unique linear equilibrium characterized by (for $n=1, \ldots, N$ ):

$$
\begin{aligned}
X_{n}\left(p_{n} ; s_{i}, p^{n-1}\right) & =a\left(s_{i}-p_{n}\right), \\
Y_{n}\left(p_{n} ; v, p^{n-1}\right) & =\alpha_{n}\left(v-p_{n}\right), \\
p_{n} & =\lambda_{n} \omega_{n}+p_{n-1},
\end{aligned}
$$

where $a=\tau_{\varepsilon} / \rho, \omega_{n}=A_{n}\left(v-p_{n-1}\right)+u_{n}, \lambda_{n}=\tau_{u} A_{n} / \tau_{n}, A_{n}=\mu \alpha_{n}+(1-\mu) a, \tau_{n}=\tau_{v}+\tau_{u} \sum_{t=1}^{n} A_{t}^{2}$. At stage $n$, the strategic informed trader's expected continuation profit is given by $E\left\{\pi_{n+1} \mid v, p^{n}\right\}=\mu H_{n}\left(v-p_{n}\right)^{2}+\mu \delta_{n}$. The

${ }^{35}$ It should be clear that in equilibrium $H_{n} \geq 0$. A negative $H_{n}$ means a negative strategic informed trader's expected profit, but the strategic informed trader always has the option not to trade at any time, obtaining a zero (nonrandom) profit.

${ }^{36}$ Drop the subscript on $p$. We know that $\operatorname{cov}(v, p)=\operatorname{var} p$, since $\operatorname{cov}(v-E(v \mid p), E(v \mid p))=0$, and $p=E(v \mid p)$. Therefore, $\operatorname{var}(v-p)=\operatorname{var} v+\operatorname{var} p-2 \operatorname{cov}(v, p)=\operatorname{var} v-\operatorname{var} p$. Furthermore, $\operatorname{var} v=\operatorname{var}(v \mid p)+\operatorname{var} E(v \mid p)=$ $\operatorname{var}(v \mid p)+\operatorname{var} p$ and therefore $\operatorname{var}(v-p)=\operatorname{var}(v \mid p)$.

(c) RAND 2001. 
constants $\alpha_{n}, H_{n}$, and $\delta_{n}$ are given by the solutions to the difference equation system

$$
\begin{aligned}
\alpha_{n} & =\left[\gamma_{n}-2\left(1-\gamma_{n}\right) \mu \lambda_{n} H_{n}\right] /\left[2 \gamma_{n} \mu \lambda_{n}\right] \\
H_{n} & =\left(1-\lambda_{n+1} A_{n+1}\right)^{2}\left[\gamma_{n+1} \alpha_{n+1}+\left(1-\gamma_{n+1}\right) H_{n+1}\right] \\
\delta_{n} & =\left[\gamma_{n+1} \alpha_{n+1}+\left(1-\gamma_{n+1}\right) H_{n+1}\right]\left(\lambda_{n+1} \sigma_{u}\right)^{2}+\left(1-\gamma_{n+1}\right) \delta_{n+1}
\end{aligned}
$$

subject to the boundary conditions $H_{N}=0, \delta_{N}=0,2 \mu \alpha_{N} \lambda_{N}=1$, and the second-order conditions $\lambda_{n}>0$ for all $n=1,2, \ldots, N$.

Proof. Available on request.

\section{References}

Admati, A.R. And Pfleiderer, P. "Sunshine Trading and Financial Market Equilibrium." Review of Financial Studies, Vol. 4 (1991), pp. 443-481.

Allen, F. AND Gale, D. “Stock-price Manipulation.” Review of Financial Studies, Vol. 5 (1992), pp. 503-529.

— AND GorTON, G. "Stock Price Manipulation, Market Microstructure and Asymmetric Information." European Economic Review, Vol. 36 (1992), pp. 624-630.

Aminud, Y. AND Mendelson, H. "Trading Mechanisms and Stock Returns: An Empirical Investigation." Journal of Finance, Vol. 42 (1987), pp. 533-553.

BACK , K. "Insider Trading in Continuous Time." Review of Financial Studies, Vol. 5 (1992), pp. 387-409.

BENABOU, R. AND LAROQUE, G. "Using Privileged Information to Manipulate Markets: Insiders, Gurus, and Credibility." Quarterly Journal of Economics, Vol. 107 (1992), pp. 921-958.

Biais, B., Hillion, P., And Spatt, C. "An Empirical Analysis of the Limit Order Book and the Order Flow in the Paris Bourse." Journal of Finance, Vol. 50 (1995), pp. 1655-1689.

—, , AND — . " Price Discovery and Learning in the Preopening Period in the Paris Bourse." Journal of Political Economy, Vol. 107 (1999), pp. 1218-1248.

BrunNERmeler, M. "Buy on Rumors-Sell on News: A Manipulative Trading Strategy." Mimeo, London School of Economics, 1998.

CAMERER, C.F. "Can Asset Markets Be Manipulated? A Field Experiment with Racetrack Betting." Journal of Political Economy, Vol. 106 (1998), pp. 457-482.

CaO, C.H., Ghysels, E., And Hatheway, F.M. "Why Is the Bid Price Greater Than the Ask? Price Discovery During the Nasdaq Pre-Opening." Mimeo, 1998.

Chan, L.K.-C. AND LAKonishoK, J. "The Behavior of Stock Prices Around Institutional Trades." Journal of Finance, Vol. 50 (1995), pp. 1147-1174.

ECONOMides, N. AND SchwarTZ, R.A. "Electronic Call Market Trading." Journal of Portfolio Management, Vol. 21 (1995), pp. 10-118.

Fishman, M.J. AND HagerTy, K.M. "The Mandatory Disclosure of Trades and Market Liquidity." Review of Financial Studies, Vol. 8 (1995), pp. 637-676.

Foster, F.D. AND Viswanathan, S. "The Effect of Public Information and Competition on Trading Volume and Price Volatility." Review of Financial Studies, Vol. 6 (1993), pp. 23-56.

— AND — . "Strategic Trading with Asymmetrically Informed Traders and Long-Lived Information." Journal of Financial and Quantitative Analysis, Vol. 29 (1994), pp. 499-518.

Gould, J. AND Verrecchia, R. "The Information Content of Specialist Pricing." Journal of Political Economy, Vol. 93 (1985), pp. 66-83.

HART, O.D. "On the Profitability of Speculation." Quarterly Journal of Economics, Vol. 91 (1977), pp. 579-597.

Hill.ion, P. AND SUOMINEN, M. "Broker Manipulation.” Mimeo, INSEAD, 1998a. — AND — . "Deadline Effect on an Order Driven Market: An Analysis of the Last Trading Minute on the Paris-Bourse." Mimeo, INSEAD, 1998b.

Holden, C.W. And Subrahmanyam, A. "Long-lived Private Information and Imperfect Competition." Journal of Finance, Vol. 47 (1992), pp. 247-270.

— AND — . "Risk Aversion, Imperfect Competition, and Long-lived Information." Economics Letters, Vol. 44 (1994), pp. 181-190.

JARrow, R.A. "Market Manipulation, Bubbles, Corners, and Short Squeezes." Journal of Financial and Quantitative Analysis, Vol. 27 (1992), pp. 311-336.

JOHN, K. AND NARAYANAN, R. "Market Manipulation and the Role of Insider Trading Regulations." Journal of Business, Vol. 70 (1997), pp. 217-247.

JoRDAN, J.S. “A Dynamic Model of Expectations Equilibrium.” Journal of Economic Theory, Vol. 28 (1982), pp. 235 -254. - "Learning Rational Expectations. The Finite State Case," Journal of Economic Theory, Vol. 36 (1985), pp. 257-276.

KeIM, D.B. AND MadHaVAn, A. "Anatomy of the Trading Process: Empirical Evidence of the Behavior of Institutional Traders." Journal of Financial Economics, Vol. 37 (1995), pp. 371-398.

(c) RAND 2001. 
AND — . "The Upstairs Market for Large-block Transactions: Analysis and Measurement of Price Effects." Review of Financial Studies, Vol. 9 (1996), pp. 1-36.

KoBAYASHI, T. “A Convergence Theorem on Rational Expectations Equilibrium with Price Information.” Working Paper no. 79, Economics Series, Institute for Mathematical Studies in the Social Sciences, Stanford University, 1977.

KumaR, P. AND SEPPI, D.J. "Futures Manipulation with 'Cash Settlement.”' Journal of Finance, Vol. 47 (1992), pp. 1485-1502.

KYLE, A.S. "Continuous Auctions and Insider Trading." Econometrica, Vol. 53 (1985), pp. 1315-1335. . "Informed Speculation with Imperfect Competition." Review of Economic Studies, Vol. 56 (1989), pp. 317-356.

LAFFONT, J.-J. AND MASKIN, E.S. "The Efficient Market Hypothesis and Insider Trading on the Stock Market." Journal of Political Economy, Vol. 98 (1990), pp. 70-93.

MA, C.-T.A. AND MANOve, M. "Bargaining with Deadlines and Imperfect Player Control." Econometrica, Vol. 61 (1993), pp. 1313-1339.

Madhavan, A. and Panchapagesan, V. "Price Discovery in Auctions Markets: A Look Inside the Black Box." Review of Financial Studies, Vol. 13 (2000), pp. 627-658.

NöTH, M. AND WEBER, M. "Insider Detection in Experimental Asset Markets." Mimeo. Mannheim: Lehrstühle für Finanzwitschaft, 1996.

PlotT, C.R., WIT, J., AND YANG, W.C. "Parimutuel Betting Markets as Information Aggregation Devices: Experimental Results.” Social Science Working Paper no. 986, California Institute of Technology, 1997.

Rochet, J.-C. AND VILA, J.-L. "Insider Trading Without Normality.” Review of Economic Studies, Vol. 61 (1994), pp. $131-152$.

Stoll, H.R. AND Whaley, R.E. "Stock Market Structure and Volatility." Review of Financial Studies, Vol. 3 (1990), pp. 37-71.

Sola, P. "Eficiencia Informativa de Precios Indicativos en el Periodo de Ajuste de la Bolsa de Madrid." Master's thesis, CEMFI, no. 9909, 1999.

Thaler, R.H. AND ZiEmbra, W.T. "Parimutuel Betting Markets: Racetracks and Lotteries." Journal of Economic Perspectives, Vol. 2 (1988), pp. 161-174.

VILA, J.-L. “Simple Games of Market Manipulation.” Economics Letters, Vol. 29 (1989), pp. 21-26.

VIVES, X. "The Speed of Information Revelation in a Financial Market Mechanism." Journal of Economic Theory, Vol. 67 (1995), pp. 178-204.

WILSON, R. "Activity Rules for the Power Exchange." Report to the California Trust For Power Industry Reestructuring, 1997.

Wrтнсомв, D.K. "An International Comparison of Stock Exchange Trading Structures." In Y. Amihud, T.S.Y. Ho, and R.A. Schwartz, eds., Market Making and the Changing Structure of the Securities Industry. Lexington, Mass.: Lexington Books, 1985. 